Annals of Mathematics, 155 (2002), 317-368

\title{
Closed characteristics on compact convex hypersurfaces in $\mathbf{R}^{2 n}$
}

\author{
By Yiming Long and Chaofeng Zhu*
}

\begin{abstract}
For any given compact $C^{2}$ hypersurface $\Sigma$ in $\mathbf{R}^{2 n}$ bounding a strictly convex set with nonempty interior, in this paper an invariant $\varrho_{n}(\Sigma)$ is defined and satisfies $\varrho_{n}(\Sigma) \geq[n / 2]+1$, where $[a]$ denotes the greatest integer which is not greater than $a \in \mathbf{R}$. The following results are proved in this paper. There always exist at least $\varrho_{n}(\Sigma)$ geometrically distinct closed characteristics on $\Sigma$. If all the geometrically distinct closed characteristics on $\Sigma$ are nondegenerate, then $\varrho_{n}(\Sigma) \geq n$. If the total number of geometrically distinct closed characteristics on $\Sigma$ is finite, there exists at least an elliptic one among them, and there exist at least $\varrho_{n}(\Sigma)-1$ of them possessing irrational mean indices. If this total number is at most $2 \varrho_{n}(\Sigma)-2$, there exist at least two elliptic ones among them.
\end{abstract}

\section{Introduction and main results}

1.1. Main results. Let $\Sigma$ be a $C^{2}$-compact hypersurface in $\mathbf{R}^{2 n}$ bounding a strictly convex compact set $C$ with nonempty interior, where $\Sigma$ has a nonvanishing Gaussian curvature. In this paper we study closed characteristics on such hypersurfaces. Without loss of generality, we may assume $0 \in C$. We denote the set of all such hypersurfaces in $\mathbf{R}^{2 n}$ by $\mathcal{H}(2 n)$. For $x \in \Sigma$, let $N_{\Sigma}(x)$ be the outward normal unit vector at $x$ of $\Sigma$. We consider the dynamics problem of finding $\tau>0$ and an absolutely continuous curve $x:[0, \tau] \rightarrow \mathbf{R}^{2 n}$ such that

$$
\left\{\begin{array}{l}
\dot{x}(t)=J N_{\Sigma}(x(t)), \quad x(t) \in \Sigma, \quad \text { for all } t \in \mathbf{R}, \\
x(\tau)=x(0),
\end{array}\right.
$$

${ }^{*}$ The first author was partially supported by the 973 Program of STM, NNSF, MCME, RFDP, PMC Key Lab of EM of China, S. S. Chern Foundation, Hong Kong Qiu Shi Sci. Tech. Foundation, and CEC of Tianjin. The first author is an associate member of ICTP. The second author was partially supported by Hong Kong Qiu Shi Sci. Tech. Foundation. 
where $J=\left(\begin{array}{cc}0 & -I \\ I & 0\end{array}\right)$ is the standard symplectic matrix on $\mathbf{R}^{2 n}$. A solution $(\tau, x)$ of the problem (1.1) is called a closed characteristic on $\Sigma$. Two closed characteristics $(\tau, x)$ and $(\sigma, y)$ are geometrically distinct, if $x(\mathbf{R}) \neq y(\mathbf{R})$. We denote by $\mathcal{J}(\Sigma)$ and $\tilde{\mathcal{J}}(\Sigma)$ the set of all closed characteristics $(\tau, x)$ on $\Sigma$ with $\tau$ being the minimal period of $x$ and the set of all geometrically distinct ones respectively. For $(\tau, x) \in \mathcal{J}(\Sigma)$, we denote by $[(\tau, x)]$ the set of all elements in $\mathcal{J}(\Sigma)$ which are geometrically the same as $(\tau, x)$. ${ }^{\#} A$ denotes the total number of elements in a set $A$.

To cast the given energy problem (1.1) into a Hamiltonian version, we follow $\S$ V.3 of I. Ekeland's celebrated book [12]. Fix a $\Sigma \in \mathcal{H}(2 n)$ bounding a convex set $C$. Then the origin is in the interior of $C$. Let $j_{C}: \mathbf{R}^{2 n} \rightarrow[0,+\infty)$ be the gauge function of $C$ defined by

$$
j_{C}(0)=0 \quad \text { and } \quad j_{C}(x)=\inf \left\{\lambda>0 \mid \frac{x}{\lambda} \in C\right\} \quad \text { for } \quad x \neq 0 .
$$

Fix a constant $\alpha$ satisfying $1<\alpha<2$ in this paper. As usual we define the Hamiltonian function $H_{\alpha}: \mathbf{R}^{2 n} \rightarrow[0,+\infty)$ by

$$
H_{\alpha}(x)=j_{C}(x)^{\alpha}, \quad \text { for all } x \in \mathbf{R}^{2 n} .
$$

Then $H_{\alpha} \in C^{1}\left(\mathbf{R}^{2 n}, \mathbf{R}\right) \cap C^{2}\left(\mathbf{R}^{2 n} \backslash\{0\}, \mathbf{R}\right)$ is convex and $\Sigma=H_{\alpha}^{-1}(1)$. It is well-known that the problem (1.1) is equivalent to the following given energy problem of the Hamiltonian system

$$
\left\{\begin{array}{l}
\dot{x}(t)=J H_{\alpha}^{\prime}(x(t)), \quad H_{\alpha}(x(t))=1, \quad \text { for all } t \in \mathbf{R} . \\
x(\tau)=x(0) .
\end{array}\right.
$$

Denote by $\mathcal{J}(\Sigma, \alpha)$ the set of all solutions $(\tau, x)$ of the problem (1.4) where $\tau$ is the minimal period of $x$, and by $\tilde{\mathcal{J}}(\Sigma, \alpha)$ the set of all geometrically distinct solutions of (1.4). Note that elements in $\mathcal{J}(\Sigma)$ and $\mathcal{J}(\Sigma, \alpha)$ are one-to-one correspondent to each other.

Let $(\tau, x) \in \mathcal{J}(\Sigma, \alpha)$. The fundamental solution $\gamma_{x}:[0, \tau] \rightarrow \operatorname{Sp}(2 n)$ with $\gamma_{x}(0)=I$ of the linearized Hamiltonian system

$$
\dot{y}(t)=J H_{\alpha}^{\prime \prime}(x(t)) y(t), \quad \text { for all } t \in \mathbf{R},
$$

is called the associated symplectic path of $(\tau, x)$. The eigenvalues of $\gamma_{x}(\tau)$ are called Floquet multipliers of $(\tau, x)$. By Proposition I.6.13 of [12], the Floquet multipliers with their multiplicity and Krein signs of $(\tau, x) \in \mathcal{J}(\Sigma)$ do not depend on the particular choice of the Hamiltonian function in (1.4). For any $M \in \operatorname{Sp}(2 n)$, we define the elliptic height $e(M)$ of $M$ to be the total algebraic multiplicity of all eigenvalues of $M$ on the unit circle $\mathbf{U}=\{z \in \mathbf{C}|| z \mid=1\}$ in the complex plane $\mathbf{C}$. Since $M$ is symplectic, $e(M)$ is even, and $0 \leq e(M) \leq 2 n$. As usual a $(\tau, x) \in \mathcal{J}(\Sigma)$ is elliptic, if $e\left(\gamma_{x}(\tau)\right)=2 n$. It is nondegenerate, if 1 
is a double Floquet multiplier of it. It is hyperbolic, if 1 is a double Floquet multiplier of it, and $e\left(\gamma_{x}(\tau)\right)=2$. It is well-known that these concepts are independent of the choice of $\alpha>1$.

The study on closed characteristics in the global sense started in 1978, when the existence of at least one closed characteristic on any $\Sigma \in \mathcal{H}(2 n)$ was first established by P. Rabinowitz in [37] (for star-shaped hypersurfaces) and A. Weinstein in [44] independently. In I. Ekeland and L. Lassoued's [15], I. Ekeland and H. Hofer's [13] of 1987, and A. Szulkin's [39] of 1988, \# $\tilde{\mathcal{J}}(\Sigma) \geq 2$ was proved for any $\Sigma \in \mathcal{H}(2 n)$ when $n \geq 2$.

In [11] of I. Ekeland in 1986 and [27] of Y. Long in 1998, for any $\Sigma \in \mathcal{H}(2 n)$ the existence of at least one nonhyperbolic closed characteristic on $\Sigma$ was proved provided ${ }^{\#} \tilde{\mathcal{J}}(\Sigma)<+\infty$. In a recent paper [31] of Y. Long, it was proved that for any $\Sigma \in \mathcal{H}(4)$, if $\# \tilde{\mathcal{J}}(\Sigma)=2$, both of the two closed characteristics must be elliptic.

Let $\mathbf{N}$ denote the set of natural numbers. Our following main results in this paper generalize the above mentioned results.

Definition 1.1. For $\alpha \in(1,2)$, we define a map $\varrho_{n}: \mathcal{H}(2 n) \rightarrow \mathbf{N} \cup\{+\infty\}$ by

$$
\varrho_{n}(\Sigma)=\left\{\begin{array}{lrl}
+\infty & \text { if } \# \mathcal{V}(\Sigma, \alpha) \\
& =+\infty \\
\min \left\{\left[\frac{i(x, 1)+2 S^{+}(x)-\nu(x, 1)+n}{2}\right] \mid[(\tau, x)] \in \mathcal{V}_{\infty}(\Sigma, \alpha)\right\}, & \text { if } \# \mathcal{V}(\Sigma, \alpha) \\
& <+\infty
\end{array}\right.
$$

where $(i(x, 1), \nu(x, 1))$ is the Maslov-type index of $(\tau, x)$ defined in $\S 1.2, S^{+}(x)$ is the splitting number of $(\tau, x)$ given in Definition 1.3 , and $\mathcal{V}(\Sigma, \alpha)$ and $\mathcal{V}_{\infty}(\Sigma, \alpha)$ are given by Definition 1.4 below.

Note that when $\# \tilde{\mathcal{J}}(\Sigma)<+\infty$, the set $\mathcal{V}_{\infty}(\Sigma, \alpha)$ is nonempty and finite. By Lemma 5.1 below, $\varrho_{n}(\Sigma)$ does not depend on the choice of $\alpha \in(1,2)$ and is a shape invariant, i.e., is independent of dilations of $\Sigma$.

Theorem 1.1. For every $\Sigma \in \mathcal{H}(2 n)$,

$$
\text { \# } \tilde{\mathcal{J}}(\Sigma) \geq \varrho_{n}(\Sigma)
$$

and

$$
\varrho_{n}(\Sigma) \geq\left[\frac{n}{2}\right]+1
$$

where $[a]=\max \{k \in \mathbf{Z} \mid k \leq a\}$ for any $a \in \mathbf{R}$.

Corollary 1.1. Fix $\Sigma \in \mathcal{H}(2 n)$ and $\alpha \in(1,2)$. Suppose every $(\tau, x) \in$ $\mathcal{J}(\Sigma, \alpha)$ satisfies

$$
i(x, 1)+2 S^{+}(x)-\nu(x, 1) \geq n .
$$


Then

$$
\varrho_{n}(\Sigma) \geq n
$$

If every $(\tau, x) \in \mathcal{J}(\Sigma)$ is nondegenerate, (1.9) holds. In particular,

$$
\# \tilde{\mathcal{J}}(\Sigma) \geq n \text {. }
$$

TheOREM 1.2. For any $\Sigma \in \mathcal{H}(2 n)$ satisfying $\# \tilde{\mathcal{J}}(\Sigma)<+\infty$, there exists at least one elliptic closed characteristic on $\Sigma$.

TheOREM 1.3. For any $\Sigma \in \mathcal{H}(2 n)$ satisfying $\# \tilde{\mathcal{J}}(\Sigma)<+\infty$, there exist at least $\varrho_{n}(\Sigma)-1\left(\geq\left[\frac{n}{2}\right]\right)$ geometrically distinct closed characteristics on $\Sigma$ possessing irrational mean indices.

TheOREm 1.4. Let $\Sigma \in \mathcal{H}(2 n)$ with $n \geq 2$. Suppose

$$
\# \tilde{\mathcal{J}}(\Sigma) \leq 2 \varrho_{n}(\Sigma)-2<+\infty .
$$

Then there exist at least two elliptic elements in $\tilde{\mathcal{J}}(\Sigma)$. In particular, by (1.8) there are at least two elliptic elements in $\tilde{\mathcal{J}}(\Sigma)$ provided

$$
\# \tilde{\mathcal{J}}(\Sigma) \leq 2\left[\frac{n}{2}\right] .
$$

The study of these problems can be traced back to pioneering works [22] of A. Liapunov in 1892 and [21] of V. J. Horn in 1903. Other related significant progress can be found in [43] of A. Weinstein, [36] of J. Moser, and [3] of T. Bartsch for local results, in [12] of I. Ekeland, [8] of G. Dell'Antonio, B. D'Onofrio, and I. Ekeland, and [20] of H. Hofer, K. Wysocki, and E. Zehnder for global results, and in [14] of I. Ekeland and J.-M. Lasry, [1] of A. Ambrosetti and G. Mancini, [17] of M. Girardi, [19] of H. Hofer, and [4] of H. Berestycki, J.-M. Lasry, G. Mancini, B. Ruf for results under pinching conditions.

A typical example of $\Sigma \in \mathcal{H}(2 n)$ is the ellipsoid $\mathcal{E}_{n}(r)$ defined as follows. Let $r=\left(r_{1}, \ldots, r_{n}\right)$ with $r_{k}>0$ for $1 \leq k \leq n$. Define

$$
\mathcal{E}_{n}(r)=\left\{x=\left(x_{1}, \ldots, x_{n}\right) \in \mathbf{R}^{2 n} \mid \frac{1}{2} \sum_{k=1}^{n} \frac{\left|x_{k}\right|^{2}}{r_{k}^{2}}=1\right\} .
$$

If $r_{j} / r_{k}$ is irrational whenever $j \neq k$, this $\mathcal{E}_{n}(r)$ is called a weakly nonresonant ellipsoid. In this case there are precisely $n$ geometrically distinct closed characteristics on $\mathcal{E}_{n}(r)$, and all of them are elliptic and nondegenerate (cf. $\S I .7$ of $[12])$.

It was conjectured some time ago (cf. p.235 of [12]) that every $\Sigma \in \mathcal{H}(2 n)$ possesses always at least $n$ geometrically distinct closed characteristics. By our result, we suspect that the lower bound number $\left[\frac{n}{2}\right]+1$ found in Theorem 1.1 is the best one can hope for. We also suspect that for any $\Sigma \in \mathcal{H}(2 n)$, if 
\# $\tilde{\mathcal{J}}(\Sigma)<+\infty$, every $(\tau, x) \in \mathcal{J}(\Sigma)$ should be elliptic. This is true for $\mathcal{H}(4)$ in view of Theorem 1.6 of [31] and the above Theorem 1.4, and a result [20] by H. Hofer, K. Wysocki, and E. Zehnder which shows that $\# \tilde{\mathcal{J}}(\Sigma)<+\infty$ implies $\# \tilde{\mathcal{J}}(\Sigma)=2$ when $\Sigma \in \mathcal{H}(4)$.

In the rest of this section, we introduce the quantities used in the above theorems and describe our main ideas in their proofs. We use some ideas from [12], [27], and [31].

1.2. Maslov-type index functions and splitting numbers. As usual, the symplectic group $\operatorname{Sp}(2 n)$ is defined by

$$
\operatorname{Sp}(2 n)=\left\{M \in \mathrm{GL}(2 n, \mathbf{R}) \mid M^{T} J M=J\right\},
$$

whose topology is the one induced from that of $\mathbf{R}^{4 n^{2}}$. We are interested in paths in $\operatorname{Sp}(2 n)$ :

$$
\mathcal{P}_{\tau}(2 n)=\{\gamma \in C([0, \tau], \operatorname{Sp}(2 n)) \mid \gamma(0)=I\},
$$

which is equipped with the topology induced from that of $\operatorname{Sp}(2 n)$. The following function is introduced in [28]:

$$
D_{\omega}(M)=(-1)^{n-1} \bar{\omega}^{n} \operatorname{det}(M-\omega I), \quad \text { for all } \omega \in \mathbf{U}, M \in \operatorname{Sp}(2 n) .
$$

It is proved in [28] that this function is real. Thus for any $\omega \in \mathbf{U}$ we can define

$$
\operatorname{Sp}(2 n)_{\omega}^{0}=\left\{M \in \operatorname{Sp}(2 n) \mid D_{\omega}(M)=0\right\} .
$$

This gives a codimension- 1 hypersurface in $\operatorname{Sp}(2 n)$. For any $M \in \operatorname{Sp}(2 n)_{\omega}^{0}$, we define a co-orientation of $\operatorname{Sp}(2 n)_{\omega}^{0}$ at $M$ by the positive direction $\left.\frac{d}{d t} M e^{t \varepsilon J}\right|_{t=0}$ of the path $M e^{t \varepsilon J}$ with $0 \leq t \leq 1$ and $\varepsilon>0$ sufficiently small. We also define

$$
\begin{aligned}
\operatorname{Sp}(2 n)_{\omega}^{*} & =\operatorname{Sp}(2 n) \backslash \operatorname{Sp}(2 n)_{\omega}^{0}, \\
\mathcal{P}_{\tau, \omega}^{*}(2 n) & =\left\{\gamma \in \mathcal{P}_{\tau}(2 n) \mid \gamma(\tau) \in \operatorname{Sp}(2 n)_{\omega}^{*}\right\}, \\
\mathcal{P}_{\tau, \omega}^{0}(2 n) & =\mathcal{P}_{\tau}(2 n) \backslash \mathcal{P}_{\tau, \omega}^{*}(2 n) .
\end{aligned}
$$

For any two continuous $\operatorname{arcs} \xi$ and $\eta:[0, \tau] \rightarrow \operatorname{Sp}(2 n)$ with $\xi(\tau)=\eta(0)$, we define as usual:

$$
\eta * \xi(t)=\left\{\begin{array}{cl}
\xi(2 t), & \text { if } 0 \leq t \leq \tau / 2 \\
\eta(2 t-\tau), & \text { if } \tau / 2 \leq t \leq \tau
\end{array}\right.
$$

Given any two $2 m_{k} \times 2 m_{k}$ matrices of square block form $M_{k}=\left(\begin{array}{cc}A_{k} & B_{k} \\ C_{k} & D_{k}\end{array}\right)$ with $k=1,2$, as in [9], the $\diamond$-product of $M_{1}$ and $M_{2}$ is defined by the following $2\left(m_{1}+m_{2}\right) \times 2\left(m_{1}+m_{2}\right)$ matrix $M_{1} \diamond M_{2}$ :

$$
M_{1} \diamond M_{2}=\left(\begin{array}{cccc}
A_{1} & 0 & B_{1} & 0 \\
0 & A_{2} & 0 & B_{2} \\
C_{1} & 0 & D_{1} & 0 \\
0 & C_{2} & 0 & D_{2}
\end{array}\right) .
$$


Denote by $M^{\diamond k}$ the $k$-fold $\diamond$-product $M \diamond \cdots \diamond M$. Note that the $\diamond$-multiplication is associative, and the $\diamond$-product of any two symplectic matrices is symplectic. For any paths $\gamma_{j} \in \mathcal{P}_{\tau}\left(2 n_{j}\right)$ with $j=0$ and 1 , define $\gamma_{0} \diamond \gamma_{1}(t)=\gamma_{0}(t) \diamond \gamma_{1}(t)$ for all $t \in[0, \tau]$.

We define a special path $\xi_{n} \in \mathcal{P}_{\tau}(2 n)$ by

$$
\xi_{n}(t)=\left(\begin{array}{cc}
2-\frac{t}{\tau} & 0 \\
0 & \left(2-\frac{t}{\tau}\right)^{-1}
\end{array}\right)^{\diamond n}, \quad \text { for } 0 \leq t \leq \tau .
$$

Definition 1.2. Let $\omega \in \mathbf{U}$. For any $M \in \operatorname{Sp}(2 n)$,

$$
\nu_{\omega}(M)=\operatorname{dim}_{\mathbf{C}} \operatorname{ker}_{\mathbf{C}}(M-\omega I) .
$$

For any $\tau>0$ and $\gamma \in \mathcal{P}_{\tau}(2 n)$,

$$
\nu_{\omega}(\gamma)=\nu_{\omega}(\gamma(\tau))
$$

If $\gamma \in \mathcal{P}_{\tau, \omega}^{*}(2 n)$,

$$
i_{\omega}(\gamma)=\left[\operatorname{Sp}(2 n)_{\omega}^{0}: \gamma * \xi_{n}\right]
$$

where the right-hand side of (1.18) is the usual homotopy intersection number, and the orientation of $\gamma * \xi_{n}$ is its positive time direction under homotopy with fixed end points. If $\gamma \in \mathcal{P}_{\tau, \omega}^{0}(2 n)$, we let $\mathcal{F}(\gamma)$ be the set of all open neighborhoods of $\gamma$ in $\mathcal{P}_{\tau}(2 n)$, and define

$$
i_{\omega}(\gamma)=\sup _{U \in \mathcal{F}(\gamma)} \inf \left\{i_{\omega}(\beta) \mid \beta \in U \cap \mathcal{P}_{\tau, \omega}^{*}(2 n)\right\}
$$

Then

$$
\left(i_{\omega}(\gamma), \nu_{\omega}(\gamma)\right) \in \mathbf{Z} \times\{0,1, \ldots, 2 n\},
$$

is called the index function of $\gamma$ at $\omega$.

Note that the right-hand side of (1.19) is always finite by Proposition 4.5 and Corollary 4.6 of [26], as well as by Theorem 2.6 and Corollary 2.7 of [29].

For any symplectic path $\gamma \in \mathcal{P}_{\tau}(2 n)$ and $m \in \mathbf{N}$, we define its $m^{\text {th }}$ iteration $\gamma^{m}:[0, m \tau] \rightarrow \operatorname{Sp}(2 n)$ by

$$
\gamma^{m}(t)=\gamma(t-j \tau) \gamma(\tau)^{j}, \quad \text { for all } j \tau \leq t \leq(j+1) \tau, j=0,1, \ldots, m-1 .
$$

We still denote the extended path on $[0,+\infty)$ by $\gamma$.

Fix a $\Sigma \in \mathcal{H}(2 n)$ and a real number $\alpha \in(1,2)$. For any $(\tau, x) \in \mathcal{J}(\Sigma, \alpha)$ and $m \in \mathbf{N}$, we define its $m^{\text {th }}$ iteration $x^{m}: \mathbf{R} /(m \tau \mathbf{Z}) \rightarrow \mathbf{R}^{2 n}$ by

$$
x^{m}(t)=x(t-j \tau), \quad \text { for all } j \tau \leq t \leq(j+1) \tau, \quad j=0,1, \ldots, m-1 .
$$

We still denote by $x$ its extension to $[0,+\infty)$. 
Definition 1.3. For any $\gamma \in \mathcal{P}_{\tau}(2 n)$,

$$
(i(\gamma, m), \nu(\gamma, m))=\left(i_{1}\left(\gamma^{m}\right), \nu_{1}\left(\gamma^{m}\right)\right), \quad \text { for all } m \in \mathbf{N} .
$$

The mean index $\hat{i}(\gamma, m)$ per $m \tau$ for $m \in \mathbf{N}$ is defined by

$$
\hat{i}(\gamma, m)=\lim _{k \rightarrow+\infty} \frac{i(\gamma, m k)}{k} .
$$

For any $M \in \operatorname{Sp}(2 n)$ and $\omega \in \mathbf{U}$, we define the splitting numbers $S_{M}^{ \pm}(\omega)$ of $M$ at $\omega$ by

$$
S_{M}^{ \pm}(\omega)=\lim _{\varepsilon \rightarrow 0^{+}} i_{\omega \exp ( \pm \sqrt{-1} \varepsilon)}(\gamma)-i_{\omega}(\gamma),
$$

for any path $\gamma \in \mathcal{P}_{\tau}(2 n)$ satisfying $\gamma(\tau)=M$.

For $\Sigma \in \mathcal{H}(2 n)$ and $\alpha \in(1,2)$, let $(\tau, x) \in \mathcal{J}(\Sigma, \alpha)$. We define

$$
\begin{aligned}
S^{+}(x) & =S_{\gamma_{x}(\tau)}^{+}(1), \\
(i(x, m), \nu(x, m)) & =\left(i\left(\gamma_{x}, m\right), \nu\left(\gamma_{x}, m\right)\right), \\
\hat{i}(x, m) & =\hat{i}\left(\gamma_{x}, m\right),
\end{aligned}
$$

for all $m \in \mathbf{N}$, where $\gamma_{x}$ is the associated symplectic path of $(\tau, x)$.

As proved in [29], the mean index $\hat{i}(\gamma, m)$ is always a finite real number, and the splitting numbers topologically defined above are independent of the choice of $\gamma$ and a complete algebraic characterization of splitting numbers is given by Theorem 4.11 of [29] (i.e., Theorem 6.6 below). Note that by (5.7) of $[29]$,

$$
m \hat{i}(\gamma, 1)=\hat{i}(\gamma, m), \quad \text { for all } m \in \mathbf{N}, \gamma \in \mathcal{P}_{\tau}(2 n) .
$$

The above Maslov-type index theory $\left(i_{1}(\gamma), \nu_{1}(\gamma)\right)$ for $\gamma \in \mathcal{P}_{\tau}(2 n)$ was defined by C. Conley and E. Zehnder in [7] of 1984 when $n \geq 2$ and $\gamma \in$ $\mathcal{P}_{\tau, 1}^{*}(2 n)$, by Y. Long and E. Zehnder in [33] of 1990 when $n=1$ and $\gamma \in$ $\mathcal{P}_{\tau, 1}^{*}(2 n)$, by Y. Long in [23] and C. Viterbo [41] in 1990 independently, when $\gamma \in \mathcal{P}_{\tau, 1}^{0}(2 n)$ is the fundamental solution of some linear Hamiltonian system with continuous symmetric $\tau$-periodic coefficients, and by Y. Long in [26] of 1997 for any $\gamma \in \mathcal{P}_{\tau, 1}^{0}(2 n)$. The index function $\left(i_{\omega}(\gamma), \nu_{\omega}(\gamma)\right)$ with $\omega \in \mathbf{U}$, the Maslov-type mean index $\hat{i}(\gamma, m)$, and the splitting numbers $S_{M}^{ \pm}(\omega)$ were defined by Y. Long in [29] of 1999.

1.3. Variational setting of the problem. Fix $\Sigma \in \mathcal{H}(2 n)$ and $\alpha \in(1,2)$ for the rest of this section. To solve the given fixed energy problem (1.4) as in [12] but with our $J$ in (1.1) instead, we consider the following fixed period problem with $H_{\alpha}$ defined by (1.3):

$$
\left\{\begin{array}{l}
\dot{z}(t)=J H_{\alpha}^{\prime}(z(t)), \quad \text { for all } t \in \mathbf{R}, \\
z(1)=z(0) .
\end{array}\right.
$$


Define

$$
E=\left\{u \in L^{(\alpha-1) / \alpha}\left(\mathbf{R} / \mathbf{Z}, \mathbf{R}^{2 n}\right) \mid \int_{0}^{1} u d t=0\right\} .
$$

The corresponding Clarke-Ekeland dual action functional $f: E \rightarrow \mathbf{R}$ is defined by

$$
f(u)=\int_{0}^{1}\left\{\frac{1}{2}(J u, \Pi u)+H_{\alpha}^{*}(-J u)\right\} d t,
$$

where $\Pi u$ is defined by $\frac{d}{d t} \Pi u=u$ and $\int_{0}^{1} \Pi u d t=0$, and the usual dual function $H_{\alpha}^{*}$ of $H_{\alpha}$ is defined by

$$
H_{\alpha}^{*}(x)=\sup _{y \in \mathbf{R}^{2 n}}\left((x, y)-H_{\alpha}(y)\right) .
$$

Here $(\cdot, \cdot)$ denotes the standard inner product of $\mathbf{R}^{2 n}$. Then $f \in C^{2}(E, \mathbf{R})$.

Suppose $u \in E \backslash\{0\}$ is a critical point of $f$. By Chapter $\mathrm{V}$ of [12], there exists $\xi_{u} \in \mathbf{R}^{2 n}$ such that $z_{u}(t)=\Pi u(t)+\xi_{u}$ is a 1 -periodic solution of the problem (1.29). Let $h=H_{\alpha}\left(z_{u}(t)\right)$ and $1 / m$ be the minimal period of $z_{u}$ for some $m \in \mathbf{N}$. Define

$$
x_{u}(t)=h^{-1 / \alpha} z_{u}\left(h^{(2-\alpha) / \alpha} t\right) \quad \text { and } \quad \tau=\frac{1}{m} h^{(\alpha-2) / \alpha} .
$$

Then $x_{u}(t) \in \Sigma$ for all $t \in \mathbf{R}$ and $\left(\tau, x_{u}\right) \in \mathcal{J}(\Sigma, \alpha)$. Note that the period 1 of $z_{u}$ corresponds to the period $m \tau$ of the solution $\left(m \tau, x_{u}^{m}\right)$ of (1.4) with minimal period $\tau$.

In [10] to [12], I. Ekeland defined his Morse-type index theory for the functional $f$ at its critical points $u$. The relationship between the Ekeland index and the above Maslov-type index is given in the following lemmas.

Lemma 1.1 (cf. [6] for the nondegenerate case, Lemma 1.3 of [27] for degenerate case, and Theorem 3.2 of [34] for a different proof). For $u$ and $z_{u}$ defined as above,

$$
i\left(z_{u}, 1\right)=i_{1}^{E}(u)+n \quad \text { and } \quad \nu\left(z_{u}, 1\right)=\nu_{1}^{E}(u) .
$$

Lemma 1.2 (cf. Lemma 1.4 of [27]). For $z_{u}, x_{u}, \tau$ and $m$ defined as above,

$$
i\left(x_{u}, m\right)=i\left(z_{u}, 1\right) \quad \text { and } \quad \nu\left(x_{u}, m\right)=\nu\left(z_{u}, 1\right) .
$$

On the other hand, every solution $(\tau, x) \in \mathcal{J}(\Sigma, \alpha)$ gives rise to a sequence $\left\{z_{m}^{x}\right\}_{m \in \mathbf{N}}$ of solutions of the given period-1 problem (1.29), and a sequence $\left\{u_{m}^{x}\right\}_{m \in \mathbf{N}}$ of critical points of $f$ defined by 


$$
\begin{aligned}
z_{m}^{x}(t) & =(m \tau)^{-1 /(2-\alpha)} x(m \tau t), \\
u_{m}^{x}(t) & =(m \tau)^{(\alpha-1) /(2-\alpha)} \dot{x}(m \tau t) .
\end{aligned}
$$

Thus, from the above discussion we obtain:

Corollary 1.2 (cf. Proof of Corollary 9.4 of [9] for a direct proof). For any $(\tau, x) \in \mathcal{J}(\Sigma, \alpha)$,

$$
i(x, 1) \geq n
$$

Following $\S$ V.3 of [12], we denote by "ind" the Fadell-Rabinowitz $S^{1}$-action cohomology index theory for $S^{1}$-invariant subsets of $E$ defined in [12] (cf. [16] of E. Fadell and P. Rabinowitz for the original definition and Appendix 2 of this paper). For $[f]_{c} \equiv\{u \in E \mid f(u) \leq c\}$, the following critical values of $f$ are defined

$$
c_{k}=\inf \left\{c<0 \mid \operatorname{ind}\left([f]_{c}\right) \geq k\right\}, \quad \text { for all } k \in \mathbf{N} .
$$

Based upon [40] and Proposition 2 on p. 443 of [13], the following important result is given in Theorem V.3.4 of [12]:

Theorem 1.5. For any $k \in \mathbf{N}$, there exists $u \in E$ such that $f^{\prime}(u)=0$, $f(u)=c_{k}$, and

$$
i_{1}^{E}(u) \leq 2 k-2 \leq i_{1}^{E}(u)+\nu_{1}^{E}(u)-1 .
$$

By the Maslov-type index theory defined above, Theorem 1.5 and results of Ekeland et al. contained in (V.3.21), (V.3.22), Proposition V.3.3, and Theorem V.3.4 in the Section V.3 of [12] can be rephrased as follows in Theorem 1.6, which forms one of the bases of our proof.

THEOREM 1.6.

$$
\begin{aligned}
-\infty<c_{1} & =\inf _{u \in E} f(u) \leq c_{2} \leq \cdots \leq c_{k} \leq c_{k+1} \leq \cdots<0 \\
c_{k} & \rightarrow 0 \quad \text { as } k \rightarrow+\infty \\
\# \tilde{\mathcal{J}}(\Sigma) & =+\infty \quad \text { if } c_{k}=c_{k+1} \text { for some } k \in \mathbf{N}
\end{aligned}
$$

For any given $k \in \mathbf{N}$, there exists $(\tau, x) \in \mathcal{J}(\Sigma, \alpha)$ and $m \in \mathbf{N}$ such that for

$$
u_{m}^{x}(t)=(m \tau)^{(\alpha-1) /(2-\alpha)} \dot{x}(m \tau t), \quad 0 \leq t \leq 1,
$$

there hold

$$
\begin{aligned}
& f^{\prime}\left(u_{m}^{x}\right)=0, \quad f\left(u_{m}^{x}\right)=c_{k}, \\
& i(x, m) \leq 2 k-2+n \leq i(x, m)+\nu(x, m)-1 .
\end{aligned}
$$

Based upon (1.44) and (1.45), the following definitions are as introduced in [27]. 
Definition 1.4. For any $\Sigma \in \mathcal{H}(2 n)$ and $\alpha \in(1,2),(\tau, x) \in \mathcal{J}(\Sigma, \alpha)$ is $(m, k)$-variationally visible, if there exist some $m$ and $k \in \mathbf{N}$ such that (1.44) and (1.45) hold for $u_{m}^{x}$ defined by (1.43). We call $(\tau, x) \in \mathcal{J}(\Sigma, \alpha)$ infinite variationally visible, if there exist infinitely many $(m, k)$ such that $(\tau, x)$ is $(m, k)$-variationally visible. We denote by $\mathcal{V}(\Sigma, \alpha)$ (or $\left.\mathcal{V}_{\infty}(\Sigma, \alpha)\right)$ the subset of $\tilde{\mathcal{J}}(\Sigma, \alpha)$ in which a representative $(\tau, x) \in \mathcal{J}(\Sigma, \alpha)$ of each $[(\tau, x)]$ is variationally visible (or infinite variationally visible).

1.4. Main new ideas and sketch of proofs. We explain our ideas in the proof of Theorem 1.1 first. As in [27], we define the $m^{\text {th }}$ index interval of $(\tau, x) \in \mathcal{J}(\Sigma, \alpha)$ by the closed interval

$$
\mathcal{I}_{m}(\tau, x)=[i(x, m), i(x, m)+\nu(x, m)-1] .
$$

We call the set

$$
\mathcal{I}(\tau, x)=\bigcup_{m \geq 1} \mathcal{I}_{m}(\tau, x)
$$

the index cover set of $(\tau, x)$. In Theorem 2.3 below, the following new iteration inequality of the Maslov-type index theory is proved for any $(\tau, x) \in \mathcal{J}(\Sigma, \alpha)$ :

$$
\begin{aligned}
i(x, m+1)-i(x, m)-\nu(x, m) & \geq i(x, 1)-\frac{e\left(\gamma_{x}(\tau)\right)}{2}+1 \\
& \geq i(x, 1)-n+1, \quad \text { for all } m \in \mathbf{N} .
\end{aligned}
$$

Here we should point out that (1.48) and (1.49) always hold without the convexity condition on $\Sigma$ if we delete the 1 from the right-hand sides of these two inequalities. To get the sharper estimate with 1 , we used the convexity condition via the following splitting lemma.

Lemma 1.3 (Lemma 3.2 of [27]). Fix $\Sigma \in \mathcal{H}(2 n)$ and $\alpha \in(1,2)$. For any $(\tau, x) \in \mathcal{J}(\Sigma, \alpha)$, there exist $P \in \operatorname{Sp}(2 n)$ and $M \in \operatorname{Sp}(2 n-2)$ such that

$$
\gamma_{x}(\tau)=P^{-1}\left(N_{1}(1,1) \diamond M\right) P,
$$

where $N_{1}(1,1)=\left(\begin{array}{ll}1 & 1 \\ 0 & 1\end{array}\right)$.

The inequality (1.49) specially implies that all index intervals of $(\tau, x)$ are mutually disjoint, and introduces a way to estimate the ellipticity of $(\tau, x)$ in terms of its iterated Maslov-type indices for some $m \in \mathbf{N}$. Note that because the system (1.4) is autonomous, we always have

$$
\nu(x, m) \geq \nu(x, 1) \geq 1 .
$$

Together with Corollary 1.2 and (1.49), we then obtain

$$
\hat{i}(x, 1) \geq 2, \quad \text { for all }(\tau, x) \in \mathcal{J}(\Sigma, \alpha) .
$$


Now (1.45) can be restated as

$$
2 \mathbf{N}-2+n \subset \bigcup_{[(\tau, x)] \in \tilde{\mathcal{J}}(\Sigma, \alpha)} \mathcal{I}(\tau, x) .
$$

We call integers in the sequence $2 \mathbf{N}-2+n$ effective integers.

Now in the following we suppose

$$
\# \tilde{\mathcal{J}}(\Sigma)<+\infty
$$

Then by (1.42) no equality in (1.40) can hold, i.e., we must have

$$
-\infty<c_{1}<c_{2}<\cdots<c_{k}<c_{k+1}<\cdots<0
$$

Here we have used the multiplicity method of Fadell-Rabinowitz $S^{1}$-index theory (cf. [16]) via Theorem V.3.4 of [12]. Note that here each $c_{k}$ corresponds uniquely to an effective integer $2 k-2+n$. We observe that in this case there is a one-to-one correspondence between the effective integers and index intervals of all closed characteristics $[(\tau, x)]$ in $\mathcal{V}_{\infty}(\Sigma, \alpha)$. In other words, under the condition (1.54), an injective map $p: \mathbf{N} \rightarrow \mathcal{V}_{\infty}(\Sigma, \alpha) \times \mathbf{N}$ can be defined by (1.44), (1.45), and (1.55). We refer to Section 3 below for the precise definition of the map $p$.

From our observations on the weakly nonresonant ellipsoid as well as the study on the case of $\mathcal{H}(4)$ in [31], we noticed that in order to maximize the effect of the Fadell-Rabinowitz $S^{1}$-index theory, instead of the index interval $\mathcal{I}_{m}(\tau, x)$, we should consider the largest open interval which contains $\mathcal{I}_{m}(\tau, x)$, possesses no part of any other index interval of $(\tau, x)$, and still can be used as the target of the map $p$. This leads to our introduction of the index jump of $(\tau, x)$.

Definition 1.5. For $\Sigma \in \mathcal{H}(2 n)$ and $\alpha \in(1,2)$, we define the $m^{\text {th }}$ index jump $\mathcal{G}_{m}(\tau, x)$ of $(\tau, x) \in \mathcal{J}(\Sigma, \alpha)$ to be the open interval

$$
\mathcal{G}_{m}(\tau, x)=(i(x, m)+\nu(x, m)-1, i(x, m+2)) .
$$

When (1.54) holds, we have $\mathcal{V}_{\infty}(\Sigma, \alpha) \neq \emptyset$ and we write

$$
\mathcal{V}_{\infty}(\Sigma, \alpha)=\left\{\left[\left(\tau_{1}, x_{1}\right)\right], \ldots,\left[\left(\tau_{q}, x_{q}\right)\right]\right\} .
$$

We will show that based on the estimates (1.38) and (1.52), there are infinitely many chances that the index jumps of all the $q$ closed characteristics contain common intervals. In fact, it will be one of the important steps to show that there exist infinitely many $\left(N, m_{1}, \ldots, m_{q}\right) \in \mathbf{N}^{q+1}$ such that

$$
\emptyset \neq\left[2 N-\kappa_{1}, 2 N+\kappa_{2}\right] \subset \bigcap_{j=1}^{q} \mathcal{G}_{2 m_{j}-1}\left(\tau_{j}, x_{j}\right),
$$


where

$$
\begin{aligned}
& \kappa_{1} \equiv \kappa_{1}(\Sigma, \alpha)=\min _{1 \leq j \leq q}\left(i\left(x_{j}, 1\right)+2 S_{\gamma_{x}\left(\tau_{j}\right)}^{+}(1)-\nu\left(x_{j}, 1\right)\right) \\
& \kappa_{2} \equiv \kappa_{2}(\Sigma, \alpha)=\min _{1 \leq j \leq q}\left(i\left(x_{j}, 1\right)-1\right) .
\end{aligned}
$$

By the Fadell-Rabinowitz $S^{1}$-index theory and our above discussion on the map $p$, there is a one-to-one correspondence between the effective integers contained on the left-hand side interval of (1.58) and the index jumps on the right-hand side of it. Together with comparisons on $\kappa_{1}, \kappa_{2}$, and $\varrho_{n}(\Sigma)$, we have

$$
\begin{aligned}
q & \geq \#\left((2 \mathbf{N}-2+n) \cap\left[2 N-\kappa_{1}, 2 N+\kappa_{2}\right]\right) \\
& \geq \varrho_{n}(\Sigma) .
\end{aligned}
$$

Then by the complete understanding on the splitting numbers given in [29] (Theorem 6.6 (Appendix) below), the estimate (1.38), and the above Definition 1.1 of $\varrho_{n}(\Sigma)$, we obtain

$$
\varrho_{n}(\Sigma) \geq\left[\frac{n}{2}\right]+1
$$

This yields the results of Theorem 1.1 when we assume (1.58).

Now the existence and size of the interval $\left[2 N-\kappa_{1}, 2 N+\kappa_{2}\right]$ in (1.58) is very crucial for our multiplicity results. The proof of (1.58) depends on the new abstract precise iteration formula of the Maslov-type index theory proved in the Theorem 2.1 below for any $(\tau, x) \in \mathcal{J}(\Sigma, \alpha)$,

$$
\begin{aligned}
i(x, m)= & m\left(i(\gamma, 1)+S_{M}^{+}(1)-C(M)\right)+2 \sum_{\theta \in(0,2 \pi)} E\left(\frac{m \theta}{2 \pi}\right) S_{M}^{-}\left(e^{\sqrt{-1} \theta}\right) \\
& -\left(S_{M}^{+}(1)+C(M)\right), \quad \text { for all } m \in \mathbf{N},
\end{aligned}
$$

where $M=\gamma_{x}(\tau)$, and

$$
\begin{aligned}
E(a) & =\min \{k \in \mathbf{Z} \mid k \geq a\}, \quad \text { for all } a \in \mathbf{R}, \\
C(M) & =\sum_{0<\theta<2 \pi} S_{M}^{-}\left(e^{\sqrt{-1} \theta}\right) .
\end{aligned}
$$

Thus the change of $i(x, m)$ in $m$ consists of a linearly increasing term $m\left(i(\gamma, 1)+S_{M}^{+}(1)-C(M)\right)$, rotator terms $E\left(\frac{m \theta}{2 \pi}\right)$ with $S_{M}^{-}\left(e^{\sqrt{-1} \theta}\right)>0$, and a bounded term.

Then the control of the location and the size of the index jumps $\mathcal{G}_{2 m_{j}-1}\left(\tau_{j}, x_{j}\right)$ for $1 \leq j \leq q$ depend on the control of all the rotators in terms of the iteration time $2 m_{j}-1$ 's for $1 \leq j \leq q$. The corresponding rotators are divided into two sets according to the rotation angle $\theta /(2 \pi)$ being rational or irrational. Now we choose a large enough integer $m_{0}$ so that multiplying by 
$2 m_{0}$ makes all the rational rotation angles $2 m_{0} \theta /(2 \pi)$ become integers. Then we require that each $m_{j}$ has the form $d_{j} m_{0}$ for some $d_{j} \in \mathbf{N}$ to be determined later for $1 \leq j \leq q$. For irrational rotators, we further choose the $m_{j}$ 's so that (1.67)

$$
E\left(\frac{2 m_{j} \theta}{2 \pi}\right)-E\left(\frac{\left(2 m_{j}-1\right) \theta}{2 \pi}\right)=1 \text { or } E\left(\frac{\left(2 m_{j}+1\right) \theta}{2 \pi}\right)-E\left(\frac{2 m_{j} \theta}{2 \pi}\right)=1
$$

holds. Thus the largest jumps of irrational rotators are caught. This is realized by requiring

$$
\left\{\frac{m_{j} \theta}{\pi}\right\} \quad \text { or } \quad 1-\left\{\frac{m_{j} \theta}{\pi}\right\}
$$

to be sufficiently small, where $\{a\}=a-[a]$ for $a \in \mathbf{R}$. These requirements will imply that the index jumps $\mathcal{G}_{2 m_{j}-1}\left(\tau_{j}, x_{j}\right)$ get big enough sizes. To make them jump together we further require that all these $m_{j}$ 's with $1 \leq j \leq q$ have a common integer factor $N \in \mathbf{N}$ in some sense. By choosing this $N$ carefully we fulfill the requirements on the rational and irrational rotators, specially (1.68), simultaneously. Therefore the problem is reduced to solving the following dynamics problem on the torus. Namely, for a given $v \in \mathbf{R}^{k}$, find infinitely many $N \in \mathbf{N}$ such that the decimal part $\{N v\}$ is as close to some vertex $\chi$ of the cube $[0,1]^{k}$ as one wants; i.e., for a given small $\varepsilon>0$,

$$
|\{N v\}-\chi|<\varepsilon \text {. }
$$

We observe that the closure of $\{\{N v\} \mid N \in \mathbf{N}\}$ in the standard torus $\mathbf{T}^{k}=\mathbf{R}^{k} / \mathbf{Z}^{k}$ forms a closed additive subgroup of $\mathbf{T}^{k}$. Thus it must contain the identity element of $\mathbf{T}^{k}$. This proves the existence of the point $\chi$ and infinitely many integral $N$ 's. In Section 4 below, we prove this in such a way that the integers $\left(N, m_{1}, \ldots, m_{q}\right) \in \mathbf{N}^{q+1}$ claimed in (1.58) can be chosen simultaneously.

Here we notice that in the above arguments the convexity of $\Sigma$ is only used to get estimates (1.38), the splitting Lemma 1.3, and the following weaker version of (1.52),

$$
\hat{i}(x, 1)>0, \quad \text { for all }(\tau, x) \in \mathcal{J}(\Sigma, \alpha) .
$$

Our main idea in the proof of Theorem 1.2 is to show the existence of one closed characteristic $\left[\left(\tau_{j}, x_{j}\right)\right]$ found by Theorem 1.1 which makes both equalities hold in (1.48) and (1.49) for the chosen iteration time $m=2 m_{j}$. Then it must be elliptic. This closed characteristic is minimal according to the injection map $p$ in a certain sense.

The proof of Theorem 1.3 depends on the understanding of the mean index sequence of iterations of closed characteristics. Under the assumption (1.54), we prove in Lemma 3.1 below that according to the ordering defined 
by the injection map $p$, the corresponding mean indices of iterations of closed characteristics strictly increase; i.e.,

$$
0<\hat{i}\left(x_{j(s)}, 2 m_{j(s)}\right)<\hat{i}\left(x_{j(t)}, 2 m_{j(t)}\right),
$$

for $p(k)=\left(\left[\left(\tau_{j(k)}, x_{j(k)}\right)\right], 2 m_{j(k)}\right)$, with $k=s$ or $t$, and $1 \leq s<t \leq \varrho_{n}(\Sigma)$. Then we prove that if both mean indices $\hat{i}\left(x_{j(s)}, 1\right)$ and $\hat{i}\left(x_{j(t)}, 1\right)$ are rational, by our choice of the iteration time $m_{j}$ 's, the two iterated mean indices in (1.71) must be equal to each other. This yields a contradiction.

To prove Theorem 1.4, we further observe that the elliptic solution found in Theorem 1.2 corresponds to the vertex $\chi$ of the cube $[0,1]^{k}$ in (1.69) via the injection map $p$. By Theorem 1.3, when $n \geq 2$ there exist at least two such vertices which make (1.69) hold. Then we prove that they produce two different elliptic closed orbits.

This paper is organized as follows. In Section 2, we derive the abstract precise iteration formula (1.64) and the iteration inequality (1.49). In Section 3, we give the precise definition of the injection map $p$ from effective numbers to iterations of closed characteristics. In Section 4, we prove the common index jump theorem based on properties of the torus group and iteration properties of the Maslov-type index theory established in [29] and [31]. In Section 5, we give the proofs of Theorems 1.1 to 1.4. For the reader's convenience, we give a brief review on the Maslov-type index and its iteration theory in Section 6 . In Section 7, an appendix on the Fadell-Rabinowitz cohomology index given by John Mather is included.

\section{Iteration formula and inequalities of the Maslov-type index theory}

We refer readers to Sections 1 and 6 for a brief review on the Maslovtype index theory and its iteration theory. In the following, we shall establish an abstract precise iteration formula and new iteration inequalities for the Maslov-type index theory using notation in those two sections.

2.1. Abstract precise iteration formulae of Maslov-type indices. For any $M \in \operatorname{Sp}(2 n)$, by Lemma 4.6 of [29] on the splitting numbers defined by (1.24), $S_{M}^{-}(\omega)=0$ if $\omega \notin \sigma(M)$. Thus $C(M)=\sum_{0<\theta<2 \pi} S_{M}^{-}\left(e^{\sqrt{-1} \theta}\right)$ defined in (1.66) is a finite sum. For any $x \in \mathbf{R}$, in addition to the function $E(x)$ given by (1.65), we further define functions $[\cdot], \phi(\cdot): \mathbf{R} \rightarrow \mathbf{Z}$ and $\{\cdot\}: \mathbf{R} \rightarrow(0,1)$ by

$$
\begin{aligned}
{[x] } & =\max \{k \in \mathbf{Z} \mid k \leq x\}, \\
\phi(x) & =E(x)-[x], \\
\{x\} & =x-[x] .
\end{aligned}
$$

Note particularly that $\phi(x)=0$ if $x \in \mathbf{Z}$, and $\phi(x)=1$ if $x \notin \mathbf{Z}$. 
Motivated by the precise iteration formula Theorem 1.3 of [31] (Theorem 6.7 below), we prove the following abstract precise iteration formula:

TheOREM 2.1. For $n \in \mathbf{N}, \tau>0$ and any path $\gamma \in \mathcal{P}_{\tau}(2 n)$, set $M=$ $\gamma(\tau)$. Extend $\gamma$ to the whole $[0,+\infty)$ by $(1.20)$. Then for any $m \in \mathbf{N}$,

$$
\begin{aligned}
i(\gamma, m)= & m\left(i(\gamma, 1)+S_{M}^{+}(1)-C(M)\right) \\
& +2 \sum_{\theta \in(0,2 \pi)} E\left(\frac{m \theta}{2 \pi}\right) S_{M}^{-}\left(e^{\sqrt{-1} \theta}\right)-\left(S_{M}^{+}(1)+C(M)\right) .
\end{aligned}
$$

Proof. Note that by Section 4 of [29], for a fixed path $\gamma$ the index $i_{\omega}(\gamma)$ is a step function in $\omega \in \mathbf{U}$ with possible jumps only at eigenvalues of $M=\gamma(\tau)$ on $\mathbf{U}$. The splitting numbers $S_{M}^{ \pm}\left(\omega_{0}\right)$ measure the jumps between $i_{\omega_{0}}(\gamma)$ and nearby $i_{\omega}(\gamma)$ from two sides of $\omega_{0}$ in $\mathbf{U}$. There, $S_{M}^{ \pm}(\omega)=0$ if $\omega \notin \sigma(M)$. Therefore for any $\omega_{0}=e^{\sqrt{-1} \theta_{0}} \in \mathbf{U}$ with $0 \leq \theta_{0}<2 \pi$, we denote by $\omega_{j}$, with $1 \leq j \leq p_{0}$, the eigenvalues of $M$ on $\mathbf{U}$ which are distributed counterclockwise from 1 to $\omega_{0}$ and located strictly between 1 and $\omega_{0}$. Then we have

$$
\begin{aligned}
i_{\omega_{0}}(\gamma) & =i(\gamma, 1)+S_{M}^{+}(1)+\sum_{j=1}^{p_{0}}\left(-S_{M}^{-}\left(\omega_{j}\right)+S_{M}^{+}\left(\omega_{j}\right)\right)-S_{M}^{-}\left(\omega_{0}\right) \\
& =i(\gamma, 1)+\sum_{0 \leq \theta<\theta_{0}} S_{M}^{+}\left(e^{\sqrt{-1} \theta}\right)-\sum_{0<\theta \leq \theta_{0}} S_{M}^{-}\left(e^{\sqrt{-1} \theta}\right) .
\end{aligned}
$$

Thus by the Bott-type formula in Appendix 1, Theorem 6.3, for any $m \in \mathbf{N}$,

$$
\begin{aligned}
i(\gamma, m)= & \sum_{\omega^{m}=1} i_{\omega}(\gamma) \\
= & i(\gamma, 1)+\sum_{k=1}^{m-1}\left(i(\gamma, 1)+\sum_{0 \leq \theta<\frac{2 k \pi}{m}} S_{M}^{+}\left(e^{\sqrt{-1} \theta}\right)-\sum_{0<\theta \leq \frac{2 k \pi}{m}} S_{M}^{-}\left(e^{\sqrt{-1} \theta}\right)\right) \\
= & i(\gamma, 1)+(m-1)\left(i(\gamma, 1)+S_{M}^{+}(1)\right) \\
& +\sum_{\theta \in(0,2 \pi)}\left(\sum_{\frac{m \theta}{2 \pi}<k \leq m-1} S_{M}^{+}\left(e^{\sqrt{-1} \theta}\right)-\sum_{\frac{m \theta}{2 \pi} \leq k \leq m-1} S_{M}^{-}\left(e^{\sqrt{-1} \theta}\right)\right) \\
(2.3) & i(\gamma, 1)+(m-1)\left(i(\gamma, 1)+S_{M}^{+}(1)\right) \\
& +\sum_{\theta \in(0,2 \pi)}\left(\left(m-1-\left[\frac{m \theta}{2 \pi}\right]\right) S_{M}^{+}\left(e^{\sqrt{-1} \theta}\right)\right. \\
& \left.\quad-\left[\frac{m(2 \pi-\theta)}{2 \pi}\right] S_{M}^{-}\left(e^{\sqrt{-1} \theta}\right)\right) .
\end{aligned}
$$


Here to get (2.3) from (2.2), we have used the fact that $[m-x]=m-[x]$ for $x \in \mathbf{Z}$ and $[m-x]=m-[x]-1$ for $x \notin \mathbf{Z}$ to count the number of $k$ 's in the given intervals.

By Lemma 4.6 of [29],

$$
S_{M}^{+}(\omega)=S_{M}^{-}(\bar{\omega}), \quad \text { for all } \omega \in \mathbf{U} .
$$

Thus by regrouping terms in (2.3) according to $S_{M}^{-}(\omega)$ with $\omega \in \mathbf{U}$, we get

$$
\begin{aligned}
i(\gamma, m)= & i(\gamma, 1)+(m-1)\left(i(\gamma, 1)+S_{M}^{+}(1)\right)+\left(m-1-2\left[\frac{m}{2}\right]\right) S_{M}^{-}(-1) \\
+ & +\sum_{\theta \in(0, \pi)}\left(\left(m-1-\left[\frac{m(2 \pi-\theta)}{2 \pi}\right]\right) S_{M}^{-}\left(e^{\sqrt{-1} \theta}\right)\right. \\
& \left.+\left(m-1-\left[\frac{m \theta}{2 \pi}\right]\right) S_{M}^{-}\left(e^{\sqrt{-1}(2 \pi-\theta)}\right)\right) \\
& -\sum_{\theta \in(0, \pi)}\left(\left[\frac{m(2 \pi-\theta)}{2 \pi}\right] S_{M}^{-}\left(e^{\sqrt{-1} \theta}\right)+\left[\frac{m \theta}{2 \pi}\right] S_{M}^{-}\left(e^{\sqrt{-1}(2 \pi-\theta)}\right)\right) \\
= & i(\gamma, 1)+(m-1)\left(i(\gamma, 1)+S_{M}^{+}(1)\right)+\left(m-2\left[\frac{m}{2}\right]-1\right) S_{M}^{-}(-1) \\
& +\sum_{\theta \in(0, \pi)}\left(\left(m-1-2\left[\frac{m \theta}{2 \pi}\right]\right) S_{M}^{-}\left(e^{\sqrt{-1}(2 \pi-\theta)}\right)\right. \\
& \left.+\left(m-1-2\left[\frac{m(2 \pi-\theta)}{2 \pi}\right]\right) S_{M}^{-}\left(e^{\sqrt{-1} \theta}\right)\right) .
\end{aligned}
$$

Since $E(x)+[y]=x+y$ if $x, y \in \mathbf{R}$ and $x+y \in \mathbf{Z}$, from (2.5) we obtain

$$
\begin{aligned}
i(\gamma, m)= & i(\gamma, 1)+(m-1)\left(i(\gamma, 1)+S_{M}^{+}(1)\right) \\
& +\left(2 E\left(\frac{m}{2}\right)-(m+1)\right) S_{M}^{-}(-1) \\
& +\sum_{\theta \in(0, \pi)}\left(\left(2 E\left(\frac{m(2 \pi-\theta)}{2 \pi}\right)-(m+1)\right) S_{M}^{-}\left(e^{\sqrt{-1}(2 \pi-\theta)}\right)\right. \\
& \left.\quad+\left(2 E\left(\frac{m \theta}{2 \pi}\right)-(m+1)\right) S_{M}^{-}\left(e^{\sqrt{-1} \theta}\right)\right) \\
= & i(\gamma, 1)+(m-1)\left(i(\gamma, 1)+S_{M}^{+}(1)\right)-(m+1) C(M) \\
& +\sum_{\theta \in(0,2 \pi)} 2 E\left(\frac{m \theta}{2 \pi}\right) S_{M}^{-}\left(e^{\sqrt{-1} \theta}\right) .
\end{aligned}
$$

Here to get (2.6), we have used the definition (1.66) of $C(M)$. This yields (2.1).

Remark 2.1. By direct verification, Theorem 2.1 coincides with Theorem 6.7 below. 
Corollary 2.1 (cf. Theorem 1.5 of [29], Corollary 5.1 of [31]). For any $\tau>0$ and $\gamma \in \mathcal{P}_{\tau}(2 n)$, set $M=\gamma(\tau)$. Now,

$$
\hat{i}(\gamma, 1) \equiv \lim _{k \rightarrow+\infty} \frac{i(\gamma, k)}{k}=i(\gamma, 1)+S_{M}^{+}(1)-C(M)+\sum_{\theta \in(0,2 \pi)} \frac{\theta}{\pi} S_{M}^{-}\left(e^{\sqrt{-1} \theta}\right) .
$$

2.2. New iteration inequalities of Maslov-type indices. Now we give new increasing estimates of the iterated Maslov-type indices by the following Theorems 2.2, 2.3 and 2.4. The proof of our main results in later sections of this paper will depend only on Theorems 2.2 and 2.3 here. The proof of Theorem 2.2 relies on the precise iteration formula in Theorem 6.7 below. This method actually gives us a way to detect and to prove or disprove whether a proposed iteration inequality of the Maslov-type index theory is correct whenever it can be reduced to an inequality of symplectic additive index terms. Theorem 2.4 is a generalization of Theorem 2.2. Our proof of Theorem 2.4 is rather different from and shorter than that of Theorem 2.2. This proof depends on special properties of the proposed inequality, the above Theorem 2.1, and complete understanding of splitting numbers given by Theorem 6.5 below.

Theorem 2.2. For $n \in \mathbf{N}, \tau>0$, and $\gamma \in \mathcal{P}_{\tau}(2 n)$, set $M=\gamma(\tau)$. We extend $\gamma$ to $[0,+\infty)$ by (1.20). Then for any $m \in \mathbf{N}$, there holds

$$
\begin{aligned}
\nu(\gamma, m)-\frac{e(M)}{2} & \leq i(\gamma, m+1)-i(\gamma, m)-i(\gamma, 1) \\
& \leq \nu(\gamma, 1)-\nu(\gamma, m+1)+\frac{e(M)}{2},
\end{aligned}
$$

where $e(M)$ is the elliptic height defined in Section 1.

Proof. Without loss of generality, we may set $\tau=1$. By Theorem 6.5, there exist $f \in C\left([0,1], \Omega^{0}(M)\right)$ and basic normal forms $M_{1}, \ldots, M_{p} \in \operatorname{Sp}(2)$ and $N_{1}, \ldots, N_{q} \in \operatorname{Sp}(4)$ such that

$$
f(0)=M, \quad f(1)=M_{1} \diamond \cdots \diamond M_{p} \diamond N_{1} \diamond \cdots \diamond N_{q} .
$$

Since $\operatorname{Sp}(2 n)$ is path connected, we can pick up paths $\xi_{i} \in \mathcal{P}_{1}(2)$ and $\eta_{j} \in \mathcal{P}_{1}(4)$ such that $\xi_{i}(1)=M_{i}$ and $\eta_{j}(1)=N_{j}$ for $1 \leq i \leq p$ and $1 \leq j \leq q$.

For $k \in \mathbf{Z}$, we define a new path $\beta \in \mathcal{P}_{1}(2 n)$ by

$$
\beta=\left\{\begin{array}{cc}
\left(\xi_{1} * \phi_{2 k \pi, 1}\right) \diamond \xi_{2} \diamond \cdots \xi_{p} \diamond \eta_{1} \diamond \cdots \diamond \eta_{q}, & \text { if } p \geq 1 \\
\left(\eta_{1} *\left(\phi_{2 k \pi, 1} \diamond I_{2}\right)\right) \diamond \eta_{2} \diamond \cdots \diamond \eta_{q}, & \text { if } p=0
\end{array}\right.
$$

where $\phi_{\theta, 1}$ is as defined in Section 6. By this construction and the definition of $f(1)$,

$$
\nu(\beta, 1)=\nu(\gamma, 1) \quad \text { and } \quad e(\beta(1))=e(f(1)) \leq e(M)
$$


Since the curve $f$ is in $\Omega^{0}(M)$, we can fix a $k \in \mathbf{Z}$ in (2.11) such that

$$
i(\beta, 1)=i(\gamma, 1) \text {. }
$$

Thus by Theorem 6.2,

$$
\beta \sim_{1} \gamma \text { on }[0,1] \text { along } f .
$$

Extending this homotopy to $[0,1] \times[0, m]$ for any $m \in \mathbf{N}$, we have

$$
\beta \sim_{1} \gamma \text { on }[0, m] \text { along } f^{m},
$$

where $f^{m}(s)=f(s)^{m}$ for any $s \in[0,1]$. Then by Theorem 6.1 , we obtain

$$
i(\beta, m)=i(\gamma, m), \quad \nu(\beta, m)=\nu(\gamma, m), \quad \text { for all } m \in \mathbf{N} .
$$

So by (2.12) and (2.16), it suffices to prove (2.8) and (2.9) for the path $\beta$.

Note that all terms in the two inequalities (2.8) and (2.9), except the elliptic height $e(\gamma(1))$, are symplectically additive and homotopy invariant. When we consider the path $\beta$, the elliptic height is also additive in terms of the decomposition of $\beta(1)=f(1)$ in (2.10). Thus by (2.10), (2.11), and Theorem 6.1, the proofs of both (2.8) and (2.9) for the path $\beta$ are reduced to those for each component path of $\beta$ in (2.11), i.e., any path in $\mathcal{P}_{1}(2)$ or $\mathcal{P}_{1}(4)$ ending at a basic normal form of symplectic matrices defined in Section 6 .

Next we continue our proof on paths ending at these normal forms in eight cases according to all the different patterns of iteration formulae.

Case 1. $\gamma \in \mathcal{P}_{\tau}(2)$ and $M \equiv \gamma(1)=N_{1}(1, b)$ with $b=1$ or 0 . In this case by Theorem 6.7 (or Theorem 3.4 of [31]), we have

$$
\begin{aligned}
e(M) & =2, \quad \nu(\gamma, 1)=2-b, \\
i(\gamma, m) & =m(i(\gamma, 1)+1)-1, \quad \nu(\gamma, m)=\nu(\gamma, 1), \quad \text { for all } m \in \mathbf{N} .
\end{aligned}
$$

Thus,

$$
\begin{aligned}
\nu(\gamma, m)-\frac{e(M)}{2} & =1-b, \\
i(\gamma, m+1)-i(\gamma, m)-i(\gamma, 1) & =1, \\
\nu(\gamma, 1)-\nu(\gamma, m+1)+\frac{e(M)}{2} & =1 .
\end{aligned}
$$

Then both (2.8) and (2.9) hold.

Here we notice that when $M=N_{1}(1,1)$, the left-hand side of the inequality (2.8) can be improved by 1 . We shall see this point in Theorem 2.3 below.

Case 2. $\gamma \in \mathcal{P}_{\tau}(2)$ and $M \equiv \gamma(1)=N_{1}(1,-1)$. In this case by Theorem 6.7 (or Theorem 3.4 of [31]), we have

$$
\begin{aligned}
e(M) & =2, \quad \nu(\gamma, 1)=1, \\
i(\gamma, m) & =m i(\gamma, 1), \quad \nu(\gamma, m)=\nu(\gamma, 1), \quad \text { for all } m \in \mathbf{N} .
\end{aligned}
$$


Thus,

$$
\begin{aligned}
\nu(\gamma, m)-\frac{e(M)}{2} & =0 \\
i(\gamma, m+1)-i(\gamma, m)-i(\gamma, 1) & =0 \\
\nu(\gamma, 1)-\nu(\gamma, m+1)+\frac{e(M)}{2} & =1 .
\end{aligned}
$$

Then both (2.8) and (2.9) hold.

Case 3. $\gamma \in \mathcal{P}_{\tau}(2)$ and $M \equiv \gamma(1)=N_{1}(-1,-b)$ with $b=1$ or 0 . In this case by Theorem 6.7 (or Theorem 3.5 of [31]), we have

$$
\begin{aligned}
e(M) & =2, \quad \nu(\gamma, 1)=0 \\
i(\gamma, m) & =m i(\gamma, 1)-\frac{1+(-1)^{m}}{2} \\
\nu(\gamma, m) & =\frac{1+(-1)^{m}}{1+b}, \quad \text { for all } m \in \mathbf{N} .
\end{aligned}
$$

Thus,

$$
\begin{aligned}
\nu(\gamma, m)-\frac{e(M)}{2} & =\frac{(-1)^{m}-b}{1+b}, \\
i(\gamma, m+1)-i(\gamma, m)-i(\gamma, 1) & =(-1)^{m}, \\
\nu(\gamma, 1)-\nu(\gamma, m+1)+\frac{e(M)}{2} & =\frac{(-1)^{m}+b}{1+b} .
\end{aligned}
$$

Then both (2.8) and (2.9) hold.

Case 4. $\gamma \in \mathcal{P}_{\tau}(2)$ and $M \equiv \gamma(1)=N_{1}(-1,1)$. In this case by Theorem 6.7 (or Theorem 3.5 of [31]), we have

$$
\begin{aligned}
e(M) & =2, \quad \nu(\gamma, 1)=0, \\
i(\gamma, m) & =m i(\gamma, 1), \quad \nu(\gamma, m)=\frac{1+(-1)^{m}}{2}, \quad \text { for all } m \in \mathbf{N} .
\end{aligned}
$$

Thus,

$$
\begin{aligned}
\nu(\gamma, m)-\frac{e(M)}{2} & =\frac{(-1)^{m}-1}{2} \leq 0, \\
i(\gamma, m+1)-i(\gamma, m)-i(\gamma, 1) & =0, \\
\nu(\gamma, 1)-\nu(\gamma, m+1)+\frac{e(M)}{2} & =\frac{1-(-1)^{m}}{2} \geq 0 .
\end{aligned}
$$

Then both (2.8) and (2.9) hold.

Case 5. $\gamma \in \mathcal{P}_{\tau}(2)$ and $M \equiv \gamma(1)=D(2)$ or $D(-2)$. In this case by Theorem 6.7 (or Theorem 3.6 of [31]), we have

$$
\begin{aligned}
e(M) & =0, \quad \nu(\gamma, 1)=0, \\
i(\gamma, m) & =m i(\gamma, 1), \quad \nu(\gamma, m)=0, \quad \text { for all } m \in \mathbf{N} .
\end{aligned}
$$


Thus,

$$
\begin{aligned}
\nu(\gamma, m)-\frac{e(M)}{2} & =0, \\
i(\gamma, m+1)-i(\gamma, m)-i(\gamma, 1) & =0, \\
\nu(\gamma, 1)-\nu(\gamma, m+1)+\frac{e(M)}{2} & =0 .
\end{aligned}
$$

Then both (2.8) and (2.9) hold.

Case 6. $\gamma \in \mathcal{P}_{\tau}(2)$ and $M \equiv \gamma(1)=R(\theta)$ for some $\theta \in(0, \pi) \cup(\pi, 2 \pi)$. In this case by Theorem 6.7 (or Theorem 3.7 of [31]), we have

$$
\begin{aligned}
e(M) & =2, \quad \nu(\gamma, 1)=0, \\
i(\gamma, m) & =m(i(\gamma, 1)-1)+2 E\left(\frac{m \theta}{2 \pi}\right)-1, \\
\nu(\gamma, m) & =2-2 \phi\left(\frac{m \theta}{2 \pi}\right), \text { for all } m \in \mathbf{N} .
\end{aligned}
$$

Thus,

$$
\begin{aligned}
\nu(\gamma, m)-\frac{e(M)}{2} & =1-2 \phi\left(\frac{m \theta}{2 \pi}\right) \\
i(\gamma, m+1)-i(\gamma, m)-i(\gamma, 1) & =2\left(E\left(\frac{(m+1) \theta}{2 \pi}\right)-E\left(\frac{m \theta}{2 \pi}\right)\right)-1 \\
\nu(\gamma, 1)-\nu(\gamma, m+1)+\frac{e(M)}{2} & =2 \phi\left(\frac{(m+1) \theta}{2 \pi}\right)-1
\end{aligned}
$$

Note that

$$
\begin{aligned}
2(E & \left.\left(\frac{(m+1) \theta}{2 \pi}\right)-E\left(\frac{m \theta}{2 \pi}\right)\right)-1 \\
& =1-2 \phi\left(\frac{m \theta}{2 \pi}\right)+2\left(E\left(\frac{(m+1) \theta}{2 \pi}\right)-\left[\frac{m \theta}{2 \pi}\right]-1\right) \\
& =2 \phi\left(\frac{(m+1) \theta}{2 \pi}\right)-1-2\left(E\left(\frac{m \theta}{2 \pi}\right)-\left[\frac{(m+1) \theta}{2 \pi}\right]\right),
\end{aligned}
$$

and that

$$
\begin{aligned}
& E\left(\frac{(m+1) \theta}{2 \pi}\right)-\left[\frac{m \theta}{2 \pi}\right]-1 \geq 0, \\
& E\left(\frac{m \theta}{2 \pi}\right)-\left[\frac{(m+1) \theta}{2 \pi}\right] \geq 0 .
\end{aligned}
$$

With (2.29)-(2.33), we obtain (2.8) and (2.9).

Case 7. $\gamma \in \mathcal{P}_{\tau}(4)$ and $M \equiv \gamma(1)=N_{2}(\omega, b)$ for some $\omega \in \mathbf{U} \backslash \mathbf{R}$ is nontrivial. In this case by Theorem 6.7 (or Theorem 4.5 of [31]), we have

$$
e(M)=4, \quad \nu(\gamma, 1)=0,
$$




$$
\begin{aligned}
i(\gamma, m) & =m i(\gamma, 1)+2 \phi\left(\frac{m \theta}{2 \pi}\right)-2, \\
\nu(\gamma, m) & =2-2 \phi\left(\frac{m \theta}{2 \pi}\right), \quad \text { for all } m \in \mathbf{N} .
\end{aligned}
$$

Thus,

$$
\begin{aligned}
\nu(\gamma, m)-\frac{e(M)}{2} & =-2 \phi\left(\frac{m \theta}{2 \pi}\right), \\
i(\gamma, m+1)-i(\gamma, m)-i(\gamma, 1) & =2 \phi\left(\frac{(m+1) \theta}{2 \pi}\right)-2 \phi\left(\frac{m \theta}{2 \pi}\right), \\
\nu(\gamma, 1)-\nu(\gamma, m+1)+\frac{e(M)}{2} & =2 \phi\left(\frac{(m+1) \theta}{2 \pi}\right) .
\end{aligned}
$$

Then both (2.8) and (2.9) hold.

Case 8. $\quad \gamma \in \mathcal{P}_{\tau}(4)$ and $M \equiv \gamma(1)=N_{2}(\omega, b)$ for some $\omega \in \mathbf{U} \backslash \mathbf{R}$ is trivial. In this case by Theorem 6.7 (or Theorem 4.6 of [31]), we have

$$
\begin{aligned}
& (2.38) e(M)=4, \quad \nu(\gamma, 1)=0 \\
& (2.39) i(\gamma, m)=m i(\gamma, 1), \quad \nu(\gamma, m)=2-2 \phi\left(\frac{m \theta}{2 \pi}\right), \quad \text { for all } m \in \mathbf{N}
\end{aligned}
$$

Thus,

$$
\begin{aligned}
\nu(\gamma, m)-\frac{e(M)}{2} & =-2 \phi\left(\frac{m \theta}{2 \pi}\right), \\
i(\gamma, m+1)-i(\gamma, m)-i(\gamma, 1) & =0, \\
\nu(\gamma, 1)-\nu(\gamma, m+1)+\frac{e(M)}{2} & =2 \phi\left(\frac{(m+1) \theta}{2 \pi}\right) .
\end{aligned}
$$

Then both (2.8) and (2.9) hold. The proof is complete.

Suggested by the study of Case 1 with $b=1$ of the proof of Theorem 2.1, we have the following result. Note that by Lemma 1.3 such a consideration is useful for the study of closed characteristics on convex hypersurfaces in $\mathbf{R}^{2 n}$.

Theorem 2.3. For $n \in \mathbf{N}, \tau>0$, and $\gamma \in \mathcal{P}_{\tau}(2 n)$, set $M=\gamma(\tau)$. Extend $\gamma$ to $[0,+\infty)$ by (1.20). Suppose that there exist $P \in \operatorname{Sp}(2 n)$ and $Q \in \operatorname{Sp}(2 n-2)$ such that

$$
M=P^{-1}\left(N_{1}(1,1) \diamond Q\right) P .
$$

Then for any $m \in \mathbf{N}$,

$$
\begin{aligned}
\nu(\gamma, m)-\frac{e(M)}{2}+1 & \leq i(\gamma, m+1)-i(\gamma, m)-i(\gamma, 1) \\
& \leq \nu(\gamma, 1)-\nu(\gamma, m+1)+\frac{e(M)}{2} .
\end{aligned}
$$


Proof. Without loss of generality, we may set $\tau=1$. Using the path $\phi_{\theta, 1}$ given in Section 6 , for $k \in \mathbf{Z}$ we define

$$
\zeta(t)=N_{1}(1, t), \quad \xi(t)=\zeta * \phi_{2 k \pi, 1}(t), \quad \text { for all } t \in[0,1] .
$$

Then by Theorems 6.1 and 6.7 below,

$$
\begin{aligned}
i(\xi, 1) & =2 k-1, \\
i(\xi, m) & =2 m k-1, \quad \nu(\xi, m)=1, \quad \text { for all } m \in \mathbf{N},
\end{aligned}
$$

Since $\operatorname{Sp}(2 n-2)$ is path connected, there is a path $\eta \in \mathcal{P}_{1}(2 n-2)$ such that $\eta(1)=Q$. By (2.40), we can fix a $k \in \mathbf{Z}$ so that

$$
2 k-1+i(\eta, 1)=i(\xi, 1)+i(\eta, 1)=i(\gamma, 1) .
$$

Pick a path $g \in \mathcal{P}_{1}(2 n)$ such that $g(1)=P$. Define

$$
h(s)=g(s)^{-1} M g(s), \quad \text { for all } t \in[0,1] .
$$

Then $h$ connects $M=\gamma(1)$ to $N_{1}(1,1) \diamond Q=(\xi \diamond \eta)(1)$ within $\Omega^{0}(M)$. Thus by Theorem 6.2 below,

$$
\gamma \sim_{1}(\xi \diamond \eta) \text { on }[0,1] \text { along } h .
$$

By extending this homotopy map to $[0,1] \times[0, m]$ via iteration, we obtain

$$
\gamma \sim_{1}(\xi \diamond \eta) \text { on }[0, m] \text { along } h^{m},
$$

where $h^{m}(s)=h(s)^{m}$. Therefore by Theorem 6.1 below and (2.45), for any $m \in \mathbf{N}$ we obtain

$$
\begin{aligned}
e(M) & =e\left(N_{1}(1,1)\right)+e(Q)=2+e(Q), \\
i(\gamma, m) & =i(\xi, m)+i(\eta, m)=2 m k-1+i(\eta, m), \\
\nu(\gamma, m) & =\nu(\xi, m)+\nu(\eta, m)=1+\nu(\eta, m) .
\end{aligned}
$$

Thus plugging (2.47)-(2.49) into (2.41) and (2.42), they become

$$
\begin{aligned}
\nu(\eta, m)-\frac{e(Q)}{2} & \leq i(\eta, m+1)-i(\eta, m)-i(\eta, 1) \\
& \leq \nu(\eta, 1)-\nu(\eta, m+1)+\frac{e(Q)}{2},
\end{aligned}
$$

which follows from Theorem 2.2 for the path $\eta$.

We start our generalization of Theorem 2.2 from the following two lemmas.

Lemma 2.1. For any $\omega \in \mathbf{U}$ and $M \in \mathrm{Sp}(2 n)$, denote by $\left(p_{\omega}(M), q_{\omega}(M)\right)$ the Krein type of $\omega \in \sigma(M)$. Then

$$
\begin{aligned}
& 0 \leq \nu_{\omega}(M)-S_{M}^{-}(\omega) \leq p_{\omega}(M), \\
& 0 \leq \nu_{\omega}(M)-S_{M}^{+}(\omega) \leq q_{\omega}(M) .
\end{aligned}
$$


Proof. The left inequalities are proved by Corollary 4.13 of [29]. Denote by $N$ the matrix on the right-hand side of (6.3) in Appendix 1, Theorem 6.5. Then

$$
p_{\omega}(N) \leq p_{\omega}(M), \quad q_{\omega}(N) \leq q_{\omega}(M) .
$$

By Definition 6.2 of $M \approx N$ and Theorem 6.6, we have $\nu_{\omega}(N)=\nu_{\omega}(M)$, $S_{N}^{-}(\omega)=S_{M}^{-}(\omega)$, and $S_{N}^{+}(\omega)=S_{M}^{+}(\omega)$. Thus in order to prove the right inequalities of (2.50) and (2.51), it suffices to prove them when $M$ is replaced by $N$. Then by Theorems 6.5 and 6.6 below, it suffices to prove them for each basic normal form given at the beginning of Appendix 1 and listed in (6.3) below. This is a direct verification via Theorem 6.5 below and thus is left to the readers.

Lemma 2.2. Let $A \subset \mathbf{U}$ and $A^{\prime}=\mathbf{U} \backslash A$. Suppose $A$ is symmetric with respect to $\mathbf{R}$; i.e., $\omega \in A$ implies $\bar{\omega} \in A$. Then for any $M \in \operatorname{Sp}(2 n)$,

$$
\sum_{\omega \in A}\left(\nu_{\omega}(M)-S_{M}^{-}(\omega)\right)+\sum_{\omega \in A^{\prime}} S_{M}^{-}(\omega) \leq \frac{e(M)}{2} .
$$

Proof. Fix $M \in \mathrm{Sp}(2 n)$. Let $e(M, A)$ denote the total algebraic multiplicity of all eigenvalues in $A \cap \sigma(M)$. For any $\omega \in \sigma(M) \cap \mathbf{U}$, denote its Krein type by $\left(p_{\omega}(M), q_{\omega}(M)\right)$. Since $A$ is symmetric with respect to $\mathbf{R}$, so is $A^{\prime}$. Note that by the definition of the Krein type,

$$
p_{\omega}(M)=q_{\bar{\omega}}(M), \quad \text { for all } \omega \in \mathbf{U} .
$$

Therefore we have

$$
\sum_{\omega \in A} p_{\omega}(M)=\frac{e(M, A)}{2}, \quad \sum_{\omega \in A^{\prime}} q_{\omega}(M)=\frac{e\left(M, A^{\prime}\right)}{2} .
$$

Together with Lemma 2.1, this yields

$$
\sum_{\omega \in A}\left(\nu_{\omega}(M)-S_{M}^{-}(\omega)\right) \leq \frac{e(M, A)}{2} .
$$

Using Lemma 4.10 of [29] and Theorem 6.6 below,

$$
\sum_{\omega \in A^{\prime}} S_{M}^{-}(\omega) \leq \frac{e\left(M, A^{\prime}\right)}{2} .
$$

Thus (2.52) holds.

Next we can give the following generalization of Theorem 2.2.

Theorem 2.4. With $n \in \mathbf{N}, \tau>0$, and $\gamma \in \mathcal{P}_{\tau}(2 n)$, set $M=\gamma(\tau)$. Extend $\gamma$ to $[0,+\infty)$ by (1.20). Then for any $m_{1}$ and $m_{2} \in \mathbf{N}$, 


$$
\begin{aligned}
\nu\left(\gamma, m_{1}\right)+\nu(\gamma & \left.m_{2}\right)-\nu\left(\gamma,\left(m_{1}, m_{2}\right)\right)-\frac{e(M)}{2} \\
& \leq i\left(\gamma, m_{1}+m_{2}\right)-i\left(\gamma, m_{1}\right)-i\left(\gamma, m_{2}\right) \\
& \leq \frac{e(M)}{2}+\nu\left(\gamma,\left(m_{1}, m_{2}\right)\right)-\nu\left(\gamma, m_{1}+m_{2}\right),
\end{aligned}
$$

where $\left(m_{1}, m_{2}\right)$ is the greatest common divisor of $m_{1}$ and $m_{2}$.

Proof. Fixing $m_{1}$ and $m_{2} \in \mathbf{N}$, define

$$
\begin{aligned}
\Psi_{m_{1}, m_{2}}(\theta) & =E\left(\frac{\left(m_{1}+m_{2}\right) \theta}{2 \pi}\right)-E\left(\frac{m_{1} \theta}{2 \pi}\right)-E\left(\frac{m_{2} \theta}{2 \pi}\right), \quad \text { for all } \theta \in[0,2 \pi), \\
A & =\left\{\theta \in[0,2 \pi) \mid \frac{m_{1} \theta}{2 \pi} \in \mathbf{Z} \text { or } \frac{m_{2} \theta}{2 \pi} \in \mathbf{Z}\right\}, \\
B & =\left\{\theta \in[0,2 \pi) \mid \frac{\left(m_{1}+m_{2}\right) \theta}{2 \pi} \in \mathbf{Z} \text { and } \frac{m_{1} \theta}{2 \pi} \notin \mathbf{Z}\right\}, \\
D & =\left\{\theta \in[0,2 \pi) \mid \Psi_{m_{1}, m_{2}}(\theta)=0\right\}, \\
D^{\prime} & =\left\{\theta \in(0,2 \pi) \mid \Psi_{m_{1}, m_{2}}(\theta) \neq 0\right\} .
\end{aligned}
$$

Note that we always have $\Psi_{m}(\theta)=0$ or $-1,0 \in A \subset D$ and $B \subset D^{\prime}$.

By Theorem 2.1, we have

$$
\begin{aligned}
i\left(\gamma, m_{1}+\right. & \left.m_{2}\right)-i\left(\gamma, m_{1}\right)-i\left(\gamma, m_{2}\right) \\
= & \left(S_{M}^{+}(1)+C(M)\right)+2 \sum_{\theta \in(0,2 \pi)} \Psi_{m_{1}, m_{2}}(\theta) S_{M}^{-}\left(e^{\sqrt{-1} \theta}\right) \\
= & S_{M}^{-}(1)+\left(\sum_{\theta \in D \backslash\{0\}} S_{M}^{-}\left(e^{\sqrt{-1} \theta}\right)+\sum_{\theta \in D^{\prime}} S_{M}^{-}\left(e^{\sqrt{-1} \theta}\right)\right) \\
& -2 \sum_{\theta \in D^{\prime}} S_{M}^{-}\left(e^{\sqrt{-1} \theta}\right) \\
= & \sum_{\theta \in D} S_{M}^{-}\left(e^{\sqrt{-1} \theta}\right)-\sum_{\theta \in D^{\prime}} S_{M}^{-}\left(e^{\sqrt{-1} \theta}\right) .
\end{aligned}
$$

By Lemma 2.2, noting that both $A$ and $B$ are symmetric with respect to $\mathbf{R}$, we obtain

$$
\begin{aligned}
& \sum_{\theta \in A} \nu_{e^{\sqrt{-1} \theta}}(M)-\sum_{\theta \in D} S_{M}^{-}\left(e^{\sqrt{-1} \theta}\right)+\sum_{\theta \in D^{\prime}} S_{M}^{-}\left(e^{\sqrt{-1} \theta}\right) \\
& \quad \leq \sum_{\theta \in A}\left(\nu_{e^{\sqrt{-1} \theta}}(M)-S_{M}^{-}\left(e^{\sqrt{-1} \theta}\right)\right)+\sum_{\theta \in A^{\prime}} S_{M}^{-}\left(e^{\sqrt{-1} \theta}\right) \leq \frac{e(M)}{2}, \\
& \sum_{\theta \in D} S_{M}^{-}\left(e^{\sqrt{-1} \theta}\right)+\sum_{\theta \in B} \nu_{e^{\sqrt{-1} \theta}}(M)-\sum_{\theta \in D^{\prime}} S_{M}^{-}\left(e^{\sqrt{-1} \theta}\right) \\
& \leq \sum_{\theta \in B^{\prime}} S_{M}^{-}\left(e^{\sqrt{-1} \theta}\right)+\sum_{\theta \in B}\left(\nu_{e^{\sqrt{-1} \theta}}(M)-S_{M}^{-}\left(e^{\sqrt{-1} \theta}\right)\right) \leq \frac{e(M)}{2}
\end{aligned}
$$


By the definitions of $A$ and $B$, we have

$$
\begin{aligned}
\nu\left(\gamma, m_{1}\right)+\nu\left(\gamma, m_{2}\right)-\nu\left(\gamma,\left(m_{1}, m_{2}\right)\right) & =\sum_{\theta \in A} \nu_{e^{\sqrt{-1} \theta}}(\gamma), \\
\nu\left(\gamma,\left(m_{1}, m_{2}\right)\right)-\nu\left(\gamma, m_{1}+m_{2}\right) & =\sum_{\theta \in B} \nu_{e^{\sqrt{-1} \theta}}(\gamma) .
\end{aligned}
$$

By (2.56) we have

$$
\begin{aligned}
i\left(\gamma, m_{1}+\right. & \left.m_{2}\right)-i\left(\gamma, m_{1}\right)-i\left(\gamma, m_{2}\right) \\
= & \sum_{\theta \in D} S_{M}^{-}\left(e^{\sqrt{-1} \theta}\right)-\sum_{\theta \in D^{\prime}} S_{M}^{-}\left(e^{\sqrt{-1} \theta}\right) \\
= & \sum_{\theta \in A} \nu_{e^{\sqrt{-1} \theta}(M)} \\
& -\left(\sum_{\theta \in A} \nu_{e^{\sqrt{-1} \theta}}(M)-\sum_{\theta \in D} S_{M}^{-}\left(e^{\sqrt{-1} \theta}\right)+\sum_{\theta \in D^{\prime}} S_{M}^{-}\left(e^{\sqrt{-1} \theta}\right)\right) .
\end{aligned}
$$

Thus by (2.57) and (2.60), this implies (2.54).

Similarly, we have

$$
\begin{aligned}
& i\left(\gamma, m_{1}+m_{2}\right)-i\left(\gamma, m_{1}\right)-i\left(\gamma, m_{2}\right) \\
& =\sum_{\theta \in D} S_{M}^{-}\left(e^{\sqrt{-1} \theta}\right)-\sum_{\theta \in D^{\prime}} S_{M}^{-}\left(e^{\sqrt{-1} \theta}\right)=\sum_{\theta \in B} \nu_{e^{\sqrt{-1} \theta}}(M) \\
& \quad+\left(\sum_{\theta \in D} S_{M}^{-}\left(e^{\sqrt{-1} \theta}\right)+\sum_{\theta \in B} \nu_{e^{\sqrt{-1} \theta}}(M)-\sum_{\theta \in D^{\prime}} S_{M}^{-}\left(e^{\sqrt{-1} \theta}\right)\right) .
\end{aligned}
$$

Thus by (2.58) and (2.60), we obtain (2.55).

The proof is complete.

\section{The injection map $p$}

In this section, we make precise the correspondence between effective integers and iterations of closed characteristics. This correspondence is given by the map $p$ defined by the following lemma.

Lemma 3.1. Suppose $\# \tilde{\mathcal{J}}(\Sigma, \alpha)<+\infty$. Then there exist an integer $K \geq 0$ and an injection map $p: \mathbf{N}+K \rightarrow \mathcal{V}_{\infty}(\Sigma, \alpha) \times \mathbf{N}$ such that

(i) For any $k \in \mathbf{N}+K,(\tau, x) \in \mathcal{J}(\Sigma, \alpha)$ and $m \in \mathbf{N}$ satisfying $p(k)=$ $([(\tau, x)], m),(1.44)$ and $(1.45)$ hold, and

(ii) For any $k_{j} \in \mathbf{N}+K, k_{1}<k_{2},\left(\tau_{j}, x_{j}\right) \in \mathcal{J}(\Sigma, \alpha)$ satisfying $p\left(k_{j}\right)=$ $\left(\left[\left(\tau_{j}, x_{j}\right)\right], m_{j}\right)$ with $j=1,2$,

$$
\hat{i}\left(x_{1}, m_{1}\right)<\hat{i}\left(x_{2}, m_{2}\right) \text {. }
$$


Proof. (i) By Theorem V.3.4 of [12], for each $k \in \mathbf{N}$, there is a $(\tau, x) \in$ $\mathcal{J}(\Sigma, \alpha)$ such that $(\tau, x)$ is $(m, k)$-variationally visible for some $m \in \mathbf{N}$, i.e. $[(\tau, x)] \in \mathcal{V}(\Sigma, \alpha)$. We define a map $p_{1}: \mathbf{N} \rightarrow \mathcal{V}(\Sigma, \alpha) \times \mathbf{N}$ by $p_{1}(k)=$ $([(\tau, x)], m)$. Since $\tilde{\mathcal{J}}(\Sigma)<+\infty,(1.55)$ holds by Theorem V.3.4 of [12], i.e. $c_{j}<c_{k}<0$ whenever $j<k$. Thus if $\left.p_{1}(j)=p_{1}(k)=([\tau, x)], m\right)$ for some $j<k$, by (1.44), we obtain

$$
c_{j}=f\left(u_{m}^{x}\right)=c_{k} .
$$

This contradiction proves that $p_{1}$ is injective.

Since ${ }^{\#} \tilde{\mathcal{J}}(\Sigma)<+\infty$, there exists an integer $K \geq 0$ such that all critical values $c_{k+K}$ with $k \in \mathbf{N}$ come from iterations of elements in $\mathcal{V}_{\infty}(\Sigma, \alpha)$. Thus $p_{1}(k+K) \in \mathcal{V}_{\infty}(\Sigma, \alpha) \times \mathbf{N}$ for any $k \in \mathbf{N}$. We define

$$
p(k)=p_{1}(k), \quad \text { for all } k \in \mathbf{N}+K .
$$

Then $p$ is injective, and (1.44) and (1.45) hold.

(ii) By Theorem V.3.11 of [12] we have $\gamma \equiv \gamma^{-}(\Sigma)=\gamma^{+}(\Sigma)>0$, where $\gamma^{ \pm}(\Sigma)$ are defined by (V.3.62)-(V.3.65) of [12] as follows:

$$
\begin{aligned}
& \gamma^{+}(\Sigma)=C_{\alpha}^{-1} \limsup _{k \rightarrow \infty}\left(\left(-c_{k}\right)^{(2-\alpha) / \alpha} k\right)^{-1}, \\
& \gamma^{-}(\Sigma)=C_{\alpha}^{-1} \liminf _{k \rightarrow \infty}\left(\left(-c_{k}\right)^{(2-\alpha) / \alpha} k\right)^{-1},
\end{aligned}
$$

with $C_{\alpha}=\frac{4}{\alpha}\left(1-\frac{\alpha}{2}\right)^{\frac{\alpha-2}{\alpha}}$. Thus we have

$$
\lim _{k \rightarrow+\infty} k\left|c_{k}\right|^{\frac{2-\alpha}{\alpha}} C_{\alpha}=\frac{1}{\gamma}
$$

For $k \in \mathbf{N}+K$, let $p(k)=([(\tau, x)], m)$ for some $[(x, \tau)] \in \mathcal{V}_{\infty}(\Sigma, \alpha)$ and $m \in \mathbf{N}$. Then $(x, \tau)$ is $(m, k)$-variationally visible. So by (V.3.45) of [12],

$$
\begin{aligned}
c_{k} & =f\left(u_{m}^{x}\right), \\
\left|c_{k}\right|^{\frac{2-\alpha}{\alpha}} & =2\left(C_{\alpha} m A(\tau, x)\right)^{-1}=2\left(C_{\alpha} A\left(m \tau, x^{m}\right)\right)^{-1},
\end{aligned}
$$

where $A(\tau, x)=\frac{1}{2} \int_{0}^{\tau}(\dot{x}, J x) d t$. Note that by Lemma V.3.12 of [12] and (1.28), there holds

$$
\frac{\hat{i}(x, m)}{A\left(m \tau, x^{m}\right)}=\frac{\hat{i}(x, 1)}{A(\tau, x)}=\frac{1}{\gamma} .
$$

This implies

$$
\left|c_{k}\right|^{\frac{2-\alpha}{\alpha}} \hat{i}(x, m)=\frac{2}{\gamma C_{\alpha}} .
$$

Since $c_{k}<c_{k+1}<0$ for $k \in \mathbf{N}$, we get our results. 
By Theorem 2.3 and Corollary 1.2 we have

Corollary 3.1. Fix $\Sigma \in \mathcal{H}(2 n)$ and $\alpha \in(1,2)$. For any $(\tau, x) \in \mathcal{J}(\Sigma, \alpha)$ and $m \in \mathbf{N}$,

$$
\begin{aligned}
i(x, m+1)-i(x, m) & \geq 2 \\
i(x, m+1)+\nu(x, m+1)-1 & \geq i(x, m+1)>i(x, m)+\nu(x, m)-1 . \\
\hat{i}(x, 1) & \geq 2 .
\end{aligned}
$$

Proof. It suffices to prove (3.6). By (3.4) and an induction argument, we obtain

$$
\frac{i(x, m)}{m} \geq \frac{i(x, 1)+2 m-2}{m}, \quad \text { for all } m \in \mathbf{N} .
$$

This implies (3.6).

Remark 3.1. The sharpest estimate $\hat{i}(x, 1)>2$ for every $(\tau, x) \in \mathcal{J}(\Sigma, \alpha)$ was first proved in Theorem 2 of [13] (cf. also Theorem I.7.7 of [12]). A different proof was given by Lemma 6.7 of [31]. A third proof can be given by use of Corollary 2.1 above and the study of splitting numbers in [29].

\section{The common index jump of closed characteristics}

The goal of this section is to prove the common index jump claim (1.58) as well as other related results.

\subsection{A common selection theorem.}

Theorem 4.1. Fix an integer $q>0$. Let $\mu_{i} \geq 0$ and $\beta_{i}$ be integers for all $i=1, \ldots, q$. Let $\alpha_{i, j}$ be positive numbers for $j=1, \ldots, \mu_{i}$ and $i=1, \ldots, q$. Let $\delta \in\left(0, \frac{1}{2}\right)$ satisfying

$$
\delta \max _{1 \leq i \leq q} \mu_{i}<\frac{1}{2}
$$

Set

$$
D_{i}=\beta_{i}+\sum_{j=1}^{\mu_{i}} \alpha_{i, j}, \quad \text { for } i=1, \ldots, q
$$

Suppose

$$
D_{i}>0, \quad \text { for all } i=1, \ldots, q \text {. }
$$

Then there exist infinitely many $\left(N, m_{1}, \ldots, m_{q}\right) \in \mathbf{N}^{q+1}$ such that

$$
\begin{aligned}
m_{i} \beta_{i}+\sum_{j=1}^{\mu_{i}} E\left(m_{i} \alpha_{i, j}\right)=N+\Delta_{i}, & \text { for all } i=1, \ldots, q, \\
\min \left\{\left\{m_{i} \alpha_{i, j}\right\}, 1-\left\{m_{i} \alpha_{i, j}\right\}\right\}<\delta, & \text { for all } j=1, \ldots, \mu_{i}, i=1, \ldots, q, \\
m_{i} \alpha_{i, j} \in \mathbf{N} & \text { if } \alpha_{i, j} \in \mathbf{Q},
\end{aligned}
$$


where

$$
\Delta_{i}=\sum_{0<\left\{m_{i} \alpha_{i, j}\right\}<\delta} 1
$$

for all $i=1, \ldots, q$.

Proof. Firstly, we reduce the claims (4.4)-(4.6) to a dynamical problem on a torus. Then we prove the existence of numbers $\left(N, m_{1}, \ldots, m_{q}\right)$ by using properties of closed additive subgroups of tori. The proof is carried out in two steps.

Step 1. Reduction to a problem on the torus. We consider the left-hand side of (4.4) first. Using the function $\phi(\cdot)$ defined at the beginning of Section 2, we obtain

$$
m_{i} \beta_{i}+\sum_{j=1}^{\mu_{i}} E\left(m_{i} \alpha_{i, j}\right)=m_{i} D_{i}+\sum_{j=1}^{\mu_{i}}\left(\phi\left(m_{i} \alpha_{i, j}\right)-\left\{m_{i} \alpha_{i, j}\right\}\right) .
$$

To handle all the rational rotators, for $1 \leq i \leq q$ we require each $m_{i}$ having a factor $M \in \mathbf{N}$ such that $M \alpha_{i, j} \in \mathbf{N}$ whenever $\alpha_{i, j} \in \mathbf{Q}$ for $j=1, \ldots, \mu_{i}$ and $i=1, \ldots, q$. Let $M=1$ if no such $\alpha_{i, j}$ exists.

To get the common integer $N \in \mathbf{N}$, we replace $m_{i} D_{i}$ in (4.8) by $\frac{N}{M D_{i}} M D_{i}$. To make $m_{i}$ an integer, we subtract the decimal part of $\frac{N}{M D_{i}}$ from itself. Because of the requirement of our later torus problem and to keep more flexibility in our choice of $N$, we add a term $\chi_{i} \in\{0,1\}$ to it. This yields

$$
\begin{aligned}
m_{i} D_{i} & =\frac{N}{M D_{i}} M D_{i}-\left\{\frac{N}{M D_{i}}\right\} M D_{i}+\chi_{i} M D_{i} \\
& =N+\left(\chi_{i}-\left\{\frac{N}{M D_{i}} M D_{i}\right\}\right) M D_{i} .
\end{aligned}
$$

Here we define

$$
m_{i}=\left(\left[\frac{N}{M D_{i}}\right]+\chi_{i}\right) M, \quad \text { for all } i=1, \ldots, q,
$$

where $\chi_{i}=0$ or 1 for $1 \leq i \leq q$ will be determined later. By this choice, (4.8) becomes

$$
\begin{aligned}
m_{i} \beta_{i}+\sum_{j=1}^{\mu_{i}} E\left(m_{i} \alpha_{i, j}\right)= & N+\left(\chi_{i}-\left\{\frac{N}{M D_{i}} M D_{i}\right\}\right) M D_{i} \\
& +\sum_{j=1}^{\mu_{i}}\left(\phi\left(m_{i} \alpha_{i, j}\right)-\left\{m_{i} \alpha_{i, j}\right\}\right) .
\end{aligned}
$$

Now the claims (4.5) and (4.6) require that only the following three possibilities for each term $\left\{m_{i} \alpha_{i, j}\right\}$ can happen: 

(A) $\left\{m_{i} \alpha_{i, j}\right\}=0$ if $\alpha_{i, j} \in \mathbf{Q}$
(B) $0<\left\{m_{i} \alpha_{i, j}\right\}<\delta$ if $\alpha_{i, j} \in \mathbf{R} \backslash \mathbf{Q}$, or
(C) $1-\delta<\left\{m_{i} \alpha_{i, j}\right\}<1$ if $\alpha_{i, j} \in \mathbf{R} \backslash \mathbf{Q}$.

Note that (A) already holds by our choice of $m_{i}$ 's of (4.10). Suppose now that these three requirements are fulfilled by our choice of $m_{i}$ 's. Then we have

$$
\begin{aligned}
\sum_{j=1}^{\mu_{i}}(\phi( & \left.\left.m_{i} \alpha_{i, j}\right)-\left\{m_{i} \alpha_{i, j}\right\}\right) \\
& =\sum_{A}+\sum_{B}+\sum_{C}\left(\phi\left(m_{i} \alpha_{i, j}\right)-\left\{m_{i} \alpha_{i, j}\right\}\right) \\
& =\sum_{0<\left\{m_{i} \alpha_{i, j}\right\}<\delta} 1-\sum_{0<\left\{m_{i} \alpha_{i, j}\right\}<\delta}\left\{m_{i} \alpha_{i, j}\right\}+\sum_{0<1-\left\{m_{i} \alpha_{i, j}\right\}<\delta}\left(1-\left\{m_{i} \alpha_{i, j}\right\}\right) \\
& =\Delta_{i}-\sum_{0<\left\{m_{i} \alpha_{i, j}\right\}<\delta}\left\{m_{i} \alpha_{i, j}\right\}+\sum_{0<1-\left\{m_{i} \alpha_{i, j}\right\}<\delta}\left(1-\left\{m_{i} \alpha_{i, j}\right\}\right) .
\end{aligned}
$$

Thus (4.11) becomes

$$
\begin{aligned}
m_{i} \beta_{i}+ & \sum_{j=1}^{\mu_{i}} E\left(m_{i} \alpha_{i, j}\right) \\
= & N+\left(\chi_{i}-\left\{\frac{N}{M D_{i}} M D_{i}\right\}\right) M D_{i} \\
& +\Delta_{i}-\sum_{0<\left\{m_{i} \alpha_{i, j}\right\}<\delta}\left\{m_{i} \alpha_{i, j}\right\}+\sum_{0<1-\left\{m_{i} \alpha_{i, j}\right\}<\delta}\left(1-\left\{m_{i} \alpha_{i, j}\right\}\right) .
\end{aligned}
$$

Therefore

$$
\left|m_{i} \beta_{i}+\sum_{j=1}^{\mu_{i}} E\left(m_{i} \alpha_{i, j}\right)-N-\Delta_{i}\right| \leq\left|\chi_{i}-\left\{\frac{N}{M D_{i}}\right\}\right| M D_{i}+\mu_{i} \delta .
$$

Here, to get (4.4) by (4.1) we need

$$
\left|\chi_{i}-\left\{\frac{N}{M D_{i}}\right\}\right| M D_{i}<\frac{1}{2} .
$$

Next we estimate $\left\{m_{i} \alpha_{i, j}\right\}$. By our choice of $m_{i}$ in (4.10),

$$
\begin{aligned}
\left\{m_{i} \alpha_{i, j}\right\} & =\left\{M\left(\left[\frac{N}{M D_{i}}\right]+\chi_{i}\right) \alpha_{i, j}\right\} \\
& =\left\{\frac{N \alpha_{i, j}}{D_{i}}+\left(\chi_{i}-\left\{\frac{N}{M D_{i}}\right\}\right) M \alpha_{i, j}\right\} \\
& =\left\{A_{i, j}(N)+B_{i, j}(N)\right\}
\end{aligned}
$$


where in (4.16), we set

$$
A_{i, j}(N)=\left\{\frac{N \alpha_{i, j}}{D_{i}}\right\}-\chi_{i, j}, \quad B_{i, j}(N)=\left(\chi_{i}-\left\{\frac{N}{M D_{i}}\right\}\right) M \alpha_{i, j}
$$

and $\chi_{i, j}=0$ or 1 will be determined later.

Assume now that we can choose $N \in \mathbf{N}$ such that

$$
\begin{aligned}
\left|\left\{\frac{N \alpha_{i, j}}{D_{i}}\right\}-\chi_{i, j}\right| & =\left|A_{i, j}(N)\right|<\frac{\delta_{1}}{3}, \\
\left|\left(\chi_{i}-\left\{\frac{N}{M D_{i}}\right\}\right) M \alpha_{i, j}\right| & =\left|B_{i, j}(N)\right|<\frac{\delta_{1}}{3},
\end{aligned}
$$

for a given $\delta_{1}$ satisfying $0<\delta_{1}<\delta<1 / 2$. Then if $A_{i, j}(N)+B_{i, j}(N) \geq 0$, we obtain

$$
\left\{m_{i} \alpha_{i, j}\right\}=A_{i, j}(N)+B_{i, j}(N)<\delta_{1}<\delta .
$$

If $A_{i, j}(N)+B_{i, j}(N)<0$, we obtain

$$
\left\{m_{i} \alpha_{i, j}\right\}=A_{i, j}(N)+B_{i, j}(N)-(-1) .
$$

That is,

$$
0<1-\left\{m_{i} \alpha_{i, j}\right\}=-\left(A_{i, j}(N)+B_{i, j}(N)\right)<\delta_{1}<\delta .
$$

Therefore to prove (4.4)-(4.6), by (4.15), (4.18), and (4.19), it suffices to prove that we can choose integers $\chi_{i}$ and $\chi_{i, j}$ to be 0 or 1 and choose infinitely many integers $N \in \mathbf{N}$ such that all the quantities

$$
\left|\left\{\frac{N \alpha_{i, j}}{D_{i}}\right\}-\chi_{i, j}\right| \text { and }\left|\left\{\frac{N}{M D_{i}}\right\}-\chi_{i}\right|
$$

can be made simultaneously as small as we want.

Let $n=q+\sum_{i=1}^{q} \mu_{i}$, and

$$
v=\left(\frac{1}{M D_{1}}, \ldots, \frac{1}{M D_{q}}, \frac{\alpha_{1,1}}{D_{1}}, \frac{\alpha_{1,2}}{D_{1}}, \ldots, \frac{\alpha_{1, \mu_{1}}}{D_{1}}, \frac{\alpha_{2,1}}{D_{2}}, \ldots, \frac{\alpha_{q, \mu_{q}}}{D_{q}}\right) \in \mathbf{R}^{n} .
$$

Then the problem becomes, for any given small $\varepsilon \in(0, \min \{\delta, 1 / 3\})$, to find a vertex $\chi$ of the cube $[0,1]^{n}$ and infinitely many integers $N \in \mathbf{N}$ such that

$$
|\{N v\}-\chi|<\varepsilon .
$$

This is a problem of the dynamics on the standard torus $\mathbf{T}^{n}=\mathbf{R}^{n} / \mathbf{Z}^{n}$.

Step 2. Dynamics on a torus. To solve the problem (4.22), it suffices to note that the closure $\bar{G}$ of the set $G=\{\{m v\} \mid m \in \mathbf{Z}\}$ in $\mathbf{T}^{n}$ forms a closed additive subgroup of the torus $\mathbf{T}^{n}$. Thus $\bar{G}$ is a product of a possibly lower dimensional torus and a cyclic group, and $G$ contains the identity element of $\mathbf{T}^{n}$. 
Define a dynamical system $f: \mathbf{T}^{n} \rightarrow \mathbf{T}^{n}$ by $f(x)=x+\pi(v)$ for all $x \in \mathbf{T}^{n}$. We consider the $\alpha$ and $\omega$-limit sets of $f$ which are defined as usual by

$$
\alpha(x)=\bigcap_{N \in \mathbf{N}} \overline{\left\{f^{k}(x) \mid k \leq-N\right\}} \quad \text { and } \quad \omega(x)=\bigcap_{N \in \mathbf{N}} \overline{\left\{f^{k}(x) \mid k \geq N\right\}},
$$

for all $x \in \mathbf{T}^{n}$.

If $v \in \mathbf{Q}^{n}, G$ must be the finite cyclic group generated by $\pi(v)$. So we have $\alpha(0)=\omega(0)=G=\bar{G}$.

If $v \in \mathbf{R}^{n} \backslash \mathbf{Q}^{n}$, then we have ${ }^{\#} G=+\infty$ and hence the limit sets $\alpha(0)$ and $\omega(0)$ are nonempty. By the definition of the limit sets and the fact $G$ is an additive subgroup of $\mathbf{T}^{n}$, we have

$$
\alpha(0)+G \subset \alpha(0), \quad \omega(0)+G \subset \omega(0) .
$$

Since the limit sets are closed,

$$
\alpha(0)+\bar{G} \subset \alpha(0), \quad \omega(0)+\bar{G} \subset \omega(0) .
$$

Thus by the facts that $\alpha(0) \cup \omega(0) \subset \bar{G}$ and that $\bar{G}$ is a closed additive subgroup of $\mathbf{T}^{n}$,

$$
\alpha(0)=\omega(0)=\bar{G} .
$$

Therefore there always exist infinitely many $N \in \mathbf{N}$ such that the point $\{N v\}$ is located in the open ball in $\mathbf{T}^{n}$ centered at its identity element with radius $\varepsilon<1 / 3$. More precisely, we have proved that in the product space $X=[0,1]^{n}$, there exist one vertex $x$ of $X$ and infinitely many $N \in \mathbf{N}$ such that

$$
\{N v\} \in B_{\varepsilon}(x) \cap X,
$$

where $B_{\varepsilon}(x)=\left\{y \in \mathbf{R}^{n}|| y-x \mid<\varepsilon\right\}$. Now we define $\chi=x$, and then (4.22) holds.

The proof is complete.

In order to prove Theorem 1.4, we need to know more about the possible choices of the vector $\chi$ in Theorem 4.1. The following result gives this information.

Theorem 4.2. Fix $v=\left(v_{1}, \ldots, v_{n}\right) \in \mathbf{R}^{n}$. Let $H$ be the closure of $\{\{m v\} \mid m \in \mathbf{N}\}$ in $\mathbf{T}^{n}$ and $V=T_{0} \pi^{-1} H$ be the tangent space of $\pi^{-1} H$ at the origin in $\mathbf{R}^{n}$, where $\pi: \mathbf{R}^{n} \rightarrow \mathbf{T}^{n}$ is the projection map. Define

$$
A(v)=V \backslash \cup_{v_{k} \in \mathbf{R} \backslash \mathbf{Q}}\left\{x=\left(x_{1}, \ldots, x_{n}\right) \in V \mid x_{k}=0\right\} .
$$

Define $\psi(x)=0$ when $x \geq 0$ and $\psi(x)=1$ when $x<0$. Then for any 
$a=\left(a_{1}, \ldots, a_{n}\right) \in A(v)$, the vector

$$
\chi=\left(\psi\left(a_{1}\right), \ldots, \psi\left(a_{n}\right)\right)
$$

makes (4.22) holds for infinitely many $N \in \mathbf{N}$.

Moreover, this set $A(v)$ possesses the following properties:

(a) $A(v) \neq \emptyset$.

(b) When $v \in \mathbf{Q}^{n}$, then $V=A(v)=\{0\}$.

(c) When $v \in \mathbf{R}^{n} \backslash \mathbf{Q}^{n}$, then $\operatorname{dim} V \geq 1,0 \notin A(v) \subset V, A(v)=-A(v)$, and $A(v)$ is open in $V$.

(d) When $\operatorname{dim} V=1$, then $A(v)=V \backslash\{0\}$.

(e) When $\operatorname{dim} V \geq 2, A(v)$ is obtained from $V$ by deleting all the coordinate hyperplanes with dimension strictly smaller than $\operatorname{dim} V$ from $V$, especially $\operatorname{dim} A(v)=\operatorname{dim} V$.

Proof. If $v \in \mathbf{Q}^{n}, H$ is a cyclic subgroup of $\mathbf{T}^{n}$. We then have $V=\{0\}$. Thus $A(v)=V=\{0\}$.

Suppose $v=\left(v_{1}, \ldots, v_{n}\right) \in \mathbf{R}^{n} \backslash \mathbf{Q}^{n}$. Then $H$ is a closed additive subgroup of $\mathbf{T}^{n}$ which is a product of a torus with a cyclic subgroup of $\mathbf{T}^{n}$. By the definition of $V$, we have $\operatorname{dim} V \geq 1$. For any $a=\left(a_{1}, \ldots, a_{n}\right) \in V$,

$$
a_{i} \neq 0 \quad \text { only if } v_{i} \in \mathbf{R} \backslash \mathbf{Q} .
$$

When $v_{i} \in \mathbf{R} \backslash \mathbf{Q}$, there exists $a=\left(a_{1}, \ldots, a_{n}\right) \in V$ with $a_{i} \neq 0$. Thus for any $a \in A(v)$, the point $\chi$ defined by (4.26) gives a vertex $\chi$ of $X=[0,1]^{n}$ which is in the closure of $\{\{m v\} \mid m \in \mathbf{N}\}$ in $X$. Therefore (4.22) holds for infinitely many $N \in \mathbf{N}$ and for this $\chi$.

The claims (a) to (e) follow from this argument, and their proofs are therefore omitted.

4.2. The common index jump of symplectic paths. The following is the main result of this subsection.

Theorem 4.3. Let $\gamma_{k} \in \mathcal{P}_{\tau_{k}}(2 n)$ for $k=1, \ldots, q$ be a finite collection of symplectic paths. Let $M_{k}=\gamma\left(\tau_{k}\right)$. Extend $\gamma_{k}$ to $[0,+\infty)$ by iteration via (1.20) for $k=1, \ldots, q$. Suppose

$$
\hat{i}\left(\gamma_{k}, 1\right)>0, \quad \text { for all } k=1, \ldots, q \text {. }
$$

Then there exist infinitely many $\left(N, m_{1}, \ldots, m_{q}\right) \in \mathbf{N}^{q+1}$ such that

$$
\begin{aligned}
& \nu\left(\gamma_{k}, 2 m_{k}-1\right)=\nu\left(\gamma_{k}, 1\right), \\
& \nu\left(\gamma_{k}, 2 m_{k}+1\right)=\nu\left(\gamma_{k}, 1\right),
\end{aligned}
$$




$$
\begin{aligned}
i\left(\gamma_{k}, 2 m_{k}-1\right)+\nu\left(\gamma_{k}, 2 m_{k}-1\right) & =2 N-\left(i\left(\gamma_{k}, 1\right)+2 S_{M_{k}}^{+}(1)-\nu\left(\gamma_{k}, 1\right)\right) \\
i\left(\gamma_{k}, 2 m_{k}+1\right) & =2 N+i\left(\gamma_{k}, 1\right) \\
i\left(\gamma_{k}, 2 m_{k}\right) & \geq 2 N-\frac{e\left(M_{k}\right)}{2} \geq 2 N-n \\
i\left(\gamma_{k}, 2 m_{k}\right)+\nu\left(\gamma_{k}, 2 m_{k}\right) & \leq 2 N+\frac{e\left(M_{k}\right)}{2} \leq 2 N+n
\end{aligned}
$$

for every $k=1, \ldots, q$.

Proof. We complete the proof in four steps.

Step 1. Application of Theorem 4.1. Set

$$
\begin{aligned}
\delta_{0} & =\min _{1 \leq k \leq q}\left\{\frac{1}{2}, \frac{\theta}{2 \pi}, 1-\frac{\theta}{2 \pi} \mid \theta \in(0,2 \pi) \text { and } e^{\sqrt{-1} \theta} \in \sigma\left(M_{k}\right)\right\} \\
C_{k} & =\sum_{\theta \in(0,2 \pi)} S_{M_{k}}^{-}\left(e^{\sqrt{-1} \theta}\right) \\
\rho_{k} & =i\left(\gamma_{k}, 1\right)+S_{M_{k}}^{+}(1)-C_{k} \\
I(k, m) & =m \rho_{k}+\sum_{\theta \in(0,2 \pi)} E\left(\frac{m \theta}{\pi}\right) S_{M_{k}}^{-}\left(e^{\sqrt{-1} \theta}\right)
\end{aligned}
$$

for $k=1, \ldots, q$ and $m \in \mathbf{N}$, where $\sigma\left(M_{k}\right)$ denotes the spectrum of $M_{k}$.

By the definition of $I(k, m)$, we rewrite it as

$$
I(k, m)=m \rho_{k}+\sum_{\theta \in(0,2 \pi)} \sum_{j=1}^{S_{M_{k}}^{-}\left(e^{\sqrt{-1} \theta}\right)} E\left(\frac{m \theta}{\pi}\right) .
$$

Note that by (4.27) and Corollary 2.1, for every $k=1, \ldots, q$,

$$
0<\hat{i}\left(\gamma_{k}, 1\right)=\rho_{k}+\sum_{\theta \in(0,2 \pi)} \frac{\theta}{\pi} S_{M_{k}}^{-}\left(e^{\sqrt{-1} \theta}\right)
$$

Now in the statement of Theorem 4.1, other than the integer $q$, we set

$$
\begin{aligned}
\delta & \in\left(0, \delta_{0}\right), \quad \beta_{i}=\rho_{i}, \\
\mu_{i} & =\sum_{\theta \in(0,2 \pi)} S_{M_{i}}^{-}\left(e^{\sqrt{-1} \theta}\right), \quad D_{i}=\hat{i}\left(\gamma_{i}, 1\right), \quad \text { for all } 1 \leq i \leq q, \\
\alpha_{i, j} & =\frac{\theta_{j}}{\pi}, \quad \text { where } e^{\sqrt{-1} \theta_{j}} \in \sigma\left(M_{i}\right), \quad \text { for all } 1 \leq j \leq \mu_{i}, 1 \leq i \leq q .
\end{aligned}
$$

Note that $\mu_{i}$ is a nonnegative integer by Corollary 4.13 of [29]. 
By (4.39), the condition (4.3) of Theorem 4.1 holds. Applying this theorem to $I(k, m)$ 's for $k=1, \ldots, q$ and any $\delta \in\left(0, \delta_{0}\right)$, there exist infinitely many $\left(N, m_{1}, \ldots, m_{q}\right) \in \mathbf{N}^{q+1}$ such that

$$
\begin{aligned}
& I\left(k, m_{k}\right)=N+\Delta_{k}, \\
& \min \left\{\left\{\frac{m_{k} \theta}{\pi}\right\}, 1-\left\{\frac{m_{k} \theta}{\pi}\right\}\right\}<\delta, \quad \text { if } e^{\sqrt{-1} \theta} \in \sigma(M), \\
& \frac{m_{k} \theta}{\pi} \in \mathbf{Z}, \quad \text { if } \frac{\theta}{\pi} \in \mathbf{Q} \cap(0,2) \text { and } e^{\sqrt{-1} \theta} \in \sigma\left(M_{k}\right), \\
& \Delta_{k}=\sum_{0<\left\{m_{k} \theta / \pi\right\}<\delta} S_{M_{k}}^{-}\left(e^{\sqrt{-1} \theta}\right),
\end{aligned}
$$

for $k=1, \ldots, q$.

Step 2. Verifications of (4.28) and (4.29). Whenever $e^{\sqrt{-1} \theta} \in \sigma\left(M_{k}\right)$ and $\frac{\theta}{\pi} \in \mathbf{Q} \cap(0,2)$, by (4.44), we always have $2 m_{k} \theta \in 2 \pi \mathbf{Z}$. Thus for any such $\theta$,

$$
2 m_{k} \theta \pm \theta \notin 2 \pi \mathbf{Z}
$$

Since the change of the nullity happens only when iterations of some eigenvalues in $\mathbf{U} \backslash\{1\}$ hit 1 , we obtain

$$
\nu\left(\gamma_{k}, 2 m_{k}-1\right)=\nu\left(\gamma_{k}, 2 m_{k}+1\right)=\nu\left(\gamma_{k}, 1\right) .
$$

Thus (4.28) and (4.29) hold.

Step 3. Verifications of (4.30) and (4.31). By Theorem 2.1, (4.42), (4.28), and the definition of $I(k, m)$,

$$
\begin{aligned}
i\left(\gamma_{k}, 2 m_{k}-1\right)+\nu\left(\gamma_{k}, 2 m_{k}-1\right) & \\
= & 2 I\left(k, m_{k}\right)-\left(i\left(\gamma_{k}, 1\right)+S_{M_{k}}^{+}(1)-C_{k}\right)-\left(S_{M_{k}}^{+}(1)+C_{k}\right) \\
& -2 \sum_{\theta \in(0,2 \pi)} \xi_{-}\left(m_{k}, \theta\right) S_{M_{k}}^{-}\left(e^{\sqrt{-1} \theta}\right)+\nu\left(\gamma_{k}, 1\right) \\
= & 2\left(N+\Delta_{k}\right)-\left(i\left(\gamma_{k}, 1\right)+2 S_{M_{k}}^{+}(1)\right) \\
& -2 \sum_{\theta \in(0,2 \pi)} \xi_{-}\left(m_{k}, \theta\right) S_{M_{k}}^{-}\left(e^{\sqrt{-1} \theta}\right)+\nu\left(\gamma_{k}, 1\right),
\end{aligned}
$$

where we define

$$
\begin{aligned}
\xi_{-}\left(m_{k}, \theta\right) & =E\left(\frac{2 m_{k} \theta}{2 \pi}\right)-E\left(\frac{\left(2 m_{k}-1\right) \theta}{2 \pi}\right) \\
& =E\left(\left[\frac{m_{k} \theta}{\pi}\right]+\left\{\frac{m_{k} \theta}{\pi}\right\}\right)-E\left(\left[\frac{m_{k} \theta}{\pi}\right]+\left\{\frac{m_{k} \theta}{\pi}\right\}-\frac{\theta}{2 \pi}\right) \\
& =E\left(\left\{\frac{m_{k} \theta}{\pi}\right\}\right)-E\left(\left\{\frac{m_{k} \theta}{\pi}\right\}-\frac{\theta}{2 \pi}\right) .
\end{aligned}
$$


Note that $\xi_{-}\left(m_{k}, \theta\right)$ takes only the value 0 or 1 . To evaluate $\xi_{-}\left(m_{k}, \theta\right)$ for any $\theta \in(0,2 \pi)$ and $e^{\sqrt{-1} \theta} \in \sigma\left(M_{k}\right)$ with some $k \in\{1, \ldots, q\}$, we consider the following three cases:

Case 1. $\left\{\frac{m_{k} \theta}{\pi}\right\}=0$. By $(4.47)$ and the definition of the function $E(\cdot)$, we have $\xi_{-}\left(m_{k}, \theta\right)=0$.

Case 2. $\left\{\frac{m_{k} \theta}{\pi}\right\} \in(0, \delta)$. Since $\delta \in\left(0, \delta_{0}\right)$, by the definition (4.34) of $\delta_{0}$, we obtain

$$
\left\{\frac{m_{k} \theta}{\pi}\right\}-\frac{\theta}{2 \pi}<\delta_{0}-\frac{\theta}{2 \pi} \leq 0
$$

Thus, $\xi_{-}\left(m_{k}, \theta\right)=1$.

Case 3. $\left\{\frac{m_{k} \theta}{\pi}\right\} \geq \delta$ and $S_{M_{k}}^{-}\left(e^{\sqrt{-1} \theta}\right)>0$. By (4.43),

$$
1-\delta<\left\{\frac{m_{k} \theta}{\pi}\right\}<1
$$

must hold. Thus by the fact $\delta \in\left(0, \delta_{0}\right)$ and the definition (4.34) of $\delta_{0}$,

$$
\left\{\frac{m_{k} \theta}{\pi}\right\}-\frac{\theta}{2 \pi}>1-\delta-\frac{\theta}{2 \pi}>0 \text {. }
$$

So in this case we have $\xi_{-}\left(m_{k}, \theta\right)=0$.

Therefore only in the above case 2 , the term $\xi_{-}\left(m_{k}, \theta\right)$ makes contribution. Together with (4.46) and (4.47),

$$
\begin{aligned}
i\left(\gamma_{k}, 2 m_{k}-1\right)+\nu\left(\gamma_{k}, 2 m_{k}-1\right)= & 2\left(N+\Delta_{k}\right)-\left(i\left(\gamma_{k}, 1\right)+2 S_{M_{k}}^{+}(1)\right) \\
& -2 \Delta_{k}+\nu\left(\gamma_{k}, 1\right) \\
= & 2 N-\left(i\left(\gamma_{k}, 1\right)+2 S_{M_{k}}^{+}(1)-\nu\left(\gamma_{k}, 1\right)\right) .
\end{aligned}
$$

This proves (4.30).

Similarly,

$$
\begin{aligned}
i\left(\gamma_{k}, 2 m_{k}+1\right)= & 2 I\left(k, m_{k}\right)+\left(i\left(\gamma_{k}, 1\right)+S_{M_{k}}^{+}(1)-C_{k}\right) \\
& -\left(S_{M_{k}}^{+}(1)+C_{k}\right)+2 \sum_{\theta \in(0,2 \pi)} \xi_{+}\left(m_{k}, \theta\right) S_{M_{k}}^{-}\left(e^{\sqrt{-1} \theta}\right),
\end{aligned}
$$

where $\xi_{+}\left(m_{k}, \theta\right)=E\left(\frac{\left(2 m_{k}+1\right) \theta}{2 \pi}\right)-E\left(\frac{2 m_{k} \theta}{2 \pi}\right)$. Similar to our discussion above, $\xi_{+}\left(m_{k}, \theta\right)=1$ in cases 1 and 3 above, and $\xi_{+}\left(m_{k}, \theta\right)=0$ in case 2. Thus from (4.48) we obtain

$$
\begin{aligned}
i\left(\gamma_{k}, 2 m_{k}+1\right) & =2\left(N+\Delta_{k}\right)+\left(i\left(\gamma_{k}, 1\right)-2 C_{k}\right)+2\left(C_{k}-\Delta_{k}\right) \\
& =2 N+i\left(\gamma_{k}, 1\right) .
\end{aligned}
$$

This proves (4.31). 
Step 4. Verifications of (4.32) and (4.33). By the inequality (2.9) in Theorem 2.2 and (4.29) as well as (4.31),

$$
\begin{aligned}
i\left(\gamma_{k}, 2 m_{k}\right) & \geq i\left(\gamma_{k}, 2 m_{k}+1\right)-i\left(\gamma_{k}, 1\right)-\frac{e\left(M_{k}\right)}{2} \\
& =2 N-\frac{e\left(M_{k}\right)}{2} \\
& \geq 2 N-n .
\end{aligned}
$$

This proves (4.32).

By the inequality (2.8) in Theorem 2.2 as well as (4.31),

$$
\begin{aligned}
i\left(\gamma_{k}, 2 m_{k}\right)+\nu\left(\gamma_{k}, 2 m_{k}\right) & \leq i\left(\gamma_{k}, 2 m_{k}+1\right)-i\left(\gamma_{k}, 1\right)+\frac{e\left(M_{k}\right)}{2} \\
& =2 N+\frac{e\left(M_{k}\right)}{2} \\
& \leq 2 N+n
\end{aligned}
$$

This proves (4.33) and the proof is complete.

By Lemma 1.3, the following consideration is useful for the study of closed characteristics.

THEOREM 4.4. Under the conditions of Theorem 4.3, further suppose

$$
M_{k}=P_{k}^{-1}\left(N_{1}(1,1) \diamond G_{k}\right) P_{k}
$$

for some $P_{k} \in \operatorname{Sp}(2 n)$ and $G_{k} \in \operatorname{Sp}(2 n-2)$ holds for $1 \leq k \leq q$. Then (4.33) can be improved to

$$
i\left(\gamma_{k}, 2 m_{k}\right)+\nu\left(\gamma_{k}, 2 m_{k}\right) \leq 2 N+\frac{e\left(M_{k}\right)}{2}-1 \leq 2 N+n-1
$$

Proof. In Step 4 of the proof for Theorem 4.3, we use the inequality (2.41) in Theorem 2.3 as well as (4.31), and obtain

$$
\begin{aligned}
i\left(\gamma_{k}, 2 m_{k}\right)+\nu\left(\gamma_{k}, 2 m_{k}\right) & \leq i\left(\gamma_{k}, 2 m_{k}+1\right)-i\left(\gamma_{k}, 1\right)+\frac{e\left(M_{k}\right)}{2}-1 \\
& =2 N+\frac{e\left(M_{k}\right)}{2}-1 \\
& \leq 2 N+n-1 .
\end{aligned}
$$

This proves (4.52). 


\section{Proof of the main results}

Fix $\Sigma \in \mathcal{H}(2 n)$ and $\alpha \in(1,2)$.

Lemma 5.1. (i) $\varrho_{n}(\Sigma)$ defined by (1.6) does not depend on the choice of $\alpha \in(1,2)$.

(ii) For any $s>0$ and $\Sigma \in \mathcal{H}(2 n), \varrho_{n}(s \Sigma)=\varrho_{n}(\Sigma)$.

Proof. (i) follows from Proposition I.7.5 of [12] and from a similar proof for the index functions and splitting numbers.

(ii) Let $C$ be the convex compact set bounded by $\Sigma$. By definition,

$$
\begin{aligned}
j_{s C}(x) & =s^{-1} j_{C}(x), \\
H_{s \Sigma, \alpha}(x) & =j_{s C}(x)^{\alpha}=s^{-\alpha} H_{\Sigma, \alpha}(x) .
\end{aligned}
$$

Let $(\tau, x) \in \mathcal{J}(\Sigma, \alpha)$. Then $\tau$ is the minimal period of $x$ and $H_{\Sigma, \alpha}(x(t))=1$. Set $y(t)=s x\left(s^{-2} t\right)$. Thus $s^{2} \tau$ is the minimal period of $y$ and $H_{s \Sigma, \alpha}(y(t))=1$. Since $\dot{x}=J H_{\Sigma, \alpha}^{\prime}(x)$,

$$
\begin{aligned}
\dot{y}(t) & =s^{-1} J H_{\Sigma, \alpha}^{\prime}\left(x\left(s^{-2} t\right)\right) \\
& =s^{-\alpha} J H_{\Sigma, \alpha}^{\prime}\left(s x\left(s^{-2} t\right)\right) \\
& =J H_{s \Sigma, \alpha}^{\prime}(y(t)) .
\end{aligned}
$$

So we have $\left(s^{2} \tau, y\right) \in \mathcal{J}(s \Sigma, \alpha)$. Therefore the map from $\mathcal{J}(\Sigma, \alpha)$ to $\mathcal{J}(s \Sigma, \alpha)$ defined by $(\tau, x) \mapsto\left(s^{2} \tau, s x\left(s^{-2} t\right)\right)$ is a bijection.

Let $\gamma_{x}$ be the associated symplectic path of $(\tau, x)$. Then,

$$
\begin{aligned}
\frac{d}{d t} \gamma_{x}\left(s^{-2} t\right) & \left.=s^{-2} J H_{\Sigma, \alpha}^{\prime \prime}\left(x\left(s^{-2} t\right)\right) \gamma_{x}\left(s^{-2} t\right)\right) \\
& \left.=s^{-\alpha} J H_{\Sigma, \alpha}^{\prime \prime}\left(s x\left(s^{-2} t\right)\right) \gamma_{x}\left(s^{-2} t\right)\right) \\
& \left.=J H_{s \Sigma, \alpha}^{\prime \prime}(y(t)) \gamma_{x}\left(s^{-2} t\right)\right) .
\end{aligned}
$$

So the associated symplectic path of $\left(s^{2} \tau, y\right)$ is $\gamma_{y}(t)=\gamma_{x}\left(s^{-2} t\right)$. Hence the lemma follows.

Theorem 1.1 is contained in the following result.

Theorem 5.1. Let $\Sigma \in \mathcal{H}(2 n)$ and $1<\alpha<2$. Suppose ${ }^{\#} \tilde{\mathcal{J}}(\Sigma)<+\infty$. Then

$$
\min \left\{\# \mathcal{V}_{\infty}(\Sigma, \alpha), n\right\} \geq \varrho_{n}(\Sigma) \geq\left[\frac{n}{2}\right]+1
$$


Proof. The proof is given by the following three claims.

Claim $1 .{ }^{\#} \mathcal{V}_{\infty}(\Sigma, \alpha) \geq \varrho_{n}(\Sigma)$

By Lemma 3.1, there exist an integer $K \geq 0$ and an injection map $p: \mathbf{N}+K \rightarrow \mathcal{V}_{\infty}(\Sigma, \alpha) \times \mathbf{N}$ such that

$$
i(x, m) \leq 2 k-2+n \leq i(x, m)+\nu(x, m)-1
$$

holds for any $k \in \mathbf{N}+K,(\tau, x) \in \mathcal{J}(\Sigma, \alpha)$ and $p(k)=([(\tau, x)], m)$. Denote the elements in $\mathcal{V}_{\infty}(\Sigma, \alpha)$ by

$$
\mathcal{V}_{\infty}(\Sigma, \alpha)=\left\{\left[\left(\tau_{j}, x_{j}\right)\right] \mid j=1, \ldots, q\right\}
$$

where $\left(\tau_{j}, x_{j}\right) \in \mathcal{J}(\Sigma, \alpha)$ for $j=1, \ldots, q$. By Lemma 1.3 and (3.6) of Corollary 3.1, we can apply Theorems 4.3 and 4.4, and obtain infinitely many $\left(N, m_{1}, \ldots, m_{q}\right) \in \mathbf{N}^{q+1}$ such that

$$
\begin{aligned}
& \bigcap_{j=1}^{q} \mathcal{G}_{2 m_{j}-1}\left(\tau_{j}, x_{j}\right) \quad \supset\left[2 N-\kappa_{1}, 2 N+\kappa_{2}\right], \\
& \bigcup_{j=1}^{q} \mathcal{I}_{2 m_{j}}\left(\tau_{j}, x_{j}\right) \subset[2 N-n, 2 N-2+n],
\end{aligned}
$$

where $\kappa_{1}=\kappa_{1}(\Sigma, \alpha)$ and $\kappa_{2}=\kappa_{2}(\Sigma, \alpha)$ are defined by (1.59) and (1.60) respectively. Recall that the $m^{\text {th }}$ index interval $\mathcal{I}_{m}(\tau, x)$ and the $m^{\text {th }}$ index jump $\mathcal{G}_{m}(\tau, x)$ of $(\tau, x)$ are defined by (1.46) and (1.56) respectively. Since $\Sigma$ is strictly convex, (1.38) holds by Corollary 1.2 for every $x_{j}$ with $1 \leq j \leq q$. Specifically, this yields

$$
\kappa_{2} \geq n-1 \text {. }
$$

Set $p(N-s+1)=\left(\left[\left(\tau_{j(s)}, x_{j(s)}\right], m(s)\right)\right.$ with $j(s) \in\{1, \ldots, q\}$ and $m(s) \in \mathbf{N}$ for $s=1, \ldots, \varrho_{n}(\Sigma)$. Then by definition of the map $p$ in Lemma 3.1,

$$
i\left(x_{j(s)}, m(s)\right) \leq 2 N-2 s+n \leq i\left(x_{j(s)}, m(s)\right)+\nu\left(x_{j(s)}, m(s)\right)-1 .
$$

Because $\varrho_{n}(\Sigma)=\left[\frac{\kappa_{1}+n}{2}\right]$ and $(5.5)$,

$$
\begin{aligned}
2 N-\kappa_{1} & \leq 2 N-2\left[\frac{\kappa_{1}+n}{2}\right]+n \\
& \leq 2 N-2 s+n \\
& \leq 2 N-2+n \\
& <2 N+\kappa_{2},
\end{aligned}
$$

for $s=1, \ldots, \varrho_{n}(\Sigma)$. From the definition (1.56) of the index jump, (5.3), (5.6), 
and (5.10), we obtain

$$
\begin{aligned}
i\left(x_{j(s)}, 2 m_{j(s)}-1\right)+\nu\left(x_{j(s)}, 2 m_{j(s)}-1\right)-1 \\
\quad<2 N-\kappa_{1} \\
\leq 2 N-2 s+n \\
\leq i\left(x_{j(s)}, m(s)\right)+\nu\left(x_{j(s)}, m(s)\right)-1,
\end{aligned}
$$

and

$$
\begin{aligned}
i\left(x_{j(s)}, m(s)\right) & \leq 2 N-2 s+n \\
& <2 N+\kappa_{2}<i\left(x_{j(s)}, 2 m_{j(s)}+1\right) .
\end{aligned}
$$

Comparing (5.11), (5.12), and (3.5) of Corollary 3.1, we obtain

$$
2 m_{j(s)}-1<m(s)<2 m_{j(s)}+1 .
$$

Hence $m(s)=2 m_{j(s)}$; i.e.,

$$
p(N-s+1)=\left(\left[\left(\tau_{j(s)}, x_{j(s)}\right]\right), 2 m_{j(s)}\right), \quad \text { for } s=1, \ldots, \varrho_{n}(\Sigma) .
$$

Since the map $p$ is injective when $\# \tilde{\mathcal{J}}(\Sigma)<+\infty$, these $j(s)$ 's are mutually different for $s=1, \ldots, \varrho_{n}(\Sigma)$. Therefore,

$$
q \geq \varrho_{n}(\Sigma)
$$

This proves Claim 1.

Claim 2. $\varrho_{n}(\Sigma) \leq n$.

In fact, if $\varrho_{n}(\Sigma)=\left[\frac{\kappa_{1}+n}{2}\right]>n$, we must have $\kappa_{1} \geq n+2$. Denote by $\left(\left[\left(\tau_{j}, x_{j}\right)\right], m\right)=p(N-n)$. Then

$$
i\left(x_{j}, m\right) \leq 2(N-n)-2+n \leq i\left(x_{j}, m\right)+\nu\left(x_{j}, m\right)-1 .
$$

By (5.3),

$$
\begin{aligned}
i\left(x_{j}, 2 m_{j}-1\right)+\nu\left(x_{j}, 2 m_{j}-1\right)-1 & <2 N-\kappa_{1} \\
& \leq 2(N-n)-2+n \\
& \leq i\left(x_{j}, m\right)+\nu\left(x_{j}, m\right)-1,
\end{aligned}
$$

and by (5.4),

$$
i\left(x_{j}, m\right) \leq 2(N-n)-2+n<2 N-n \leq i\left(x, 2 m_{j}\right) .
$$

Hence by Corollary 3.1, we have $2 m_{j}-1<m<2 m_{j}$. This contradiction proves Claim 2. 
Claim 3. $\varrho_{n}(\Sigma) \geq\left[\frac{n}{2}\right]+1$.

Let $(\tau, x) \in \mathcal{J}(\Sigma, \alpha)$ and $\gamma_{x}$ be its associated symplectic path. In the following, we estimate $i(x, 1)+2 S^{+}(x)-\nu(x, 1)+n$.

By Lemma 1.3, (1.50) holds for some $P \in \operatorname{Sp}(2 n)$ and $M \in \operatorname{Sp}(2 n-2)$. By $1^{\circ}$ and $2^{\circ}$ of Corollary 4.14 of [29], the splitting numbers are constant on each homotopy component and symplectic additive. Thus by (1.50),

$$
2 S^{+}(x)-\nu(x, 1)=2 S_{N_{1}(1,1)}^{+}(1)-\nu_{1}\left(N_{1}(1,1)\right)+2 S_{M}^{+}(1)-\nu_{1}(M) .
$$

By Theorem 6.6 below,

$$
S_{N_{1}(1, a)}^{+}(1)= \begin{cases}1, & \text { if } a \geq 0 \\ 0, & \text { if } a<0 .\end{cases}
$$

Thus,

$$
2 S_{N_{1}(1, a)}^{+}(1)-\nu_{1}\left(N_{1}(1, a)\right)=a, \quad \text { for } a= \pm 1,0 .
$$

By Theorem 6.5,

$$
M \approx N_{1}(1,1)^{\diamond p_{-}} \diamond I_{2}^{\diamond p_{0}} \diamond N_{1}(1,-1)^{\diamond p_{+}} \diamond G,
$$

for some nonnegative integers $p_{-}, p_{0}$, and $p_{+}$, and some symplectic matrix $G$ satisfying $1 \notin \sigma(G)$. By (5.16) and (5.17), we then obtain

$$
2 S_{M}^{+}(1)-\nu_{1}(M) \geq p_{-}-p_{+} \geq-p_{+} \geq 1-n .
$$

From (1.38), (5.15), (5.16) with $a=1$, and (5.18),

$$
i(x, 1)+2 S^{+}(x)-\nu(x, 1)+n \geq n+1+(1-n)+n=n+2 .
$$

So Claim 3 holds and the proof of Theorem 5.1 is complete.

Next we give the proof for the nondegenerate case.

Proof of Corollary 1.1. By the definition of $\varrho_{n}(\Sigma),(1.9)$, and Theorem 5.1, we obtain (1.10).

If $(\tau, x) \in \mathcal{J}(\Sigma, \alpha)$ is nondegenerate, we must have $1 \notin \sigma(M)$ in (5.15). Thus by (5.15) and (5.16), we obtain $2 S^{+}(x)-\nu_{\tau}(x)=1$. This and (1.38) give (1.9). Now, (1.10) holds and by (1.7) this implies (1.11).

Corollary 5.1. Let $\Sigma \in \mathcal{H}(2 n)$ and $\alpha \in(1,2)$. Assume $\# \tilde{\mathcal{J}}(\Sigma)<+\infty$. Then there exists an element $[(\tau, x)] \in \mathcal{V}_{\infty}(\Sigma, \alpha)$ with $i_{\tau}(x)=n$.

Proof. We use notation introduced in the proof of Theorem 5.1. Let $\left(\left[\left(\tau_{j}, x_{j}\right)\right], m\right)=p(N+1)$. By the definition of $p$, we obtain $\left[\left(\tau_{j}, x_{j}\right)\right] \in \mathcal{V}_{\infty}(\Sigma, \alpha)$ and

$$
i\left(x_{j}, m\right) \leq 2 N+n \leq i\left(x_{j}, m\right)+\nu\left(x_{j}, m\right)-1 .
$$


Thus by (5.3) and (5.4),

$$
\begin{aligned}
i\left(x_{j}, 2 m_{j}\right)+\nu\left(x_{j}, 2 m_{j}\right)-1 & \leq 2 N-2+n \\
& <2 N+n \\
& \leq 2 N+\kappa_{2}+1 \\
& \leq i\left(x_{j}, 2 m_{j}+1\right) .
\end{aligned}
$$

Hence by Corollary 3.1, comparing (5.19) and (5.20) we obtain $2 m_{j}<m \leq$ $2 m_{j}+1$. Thus $m=2 m_{j}+1$. By (5.19) and (5.20) again,

$$
i\left(x, 2 m_{j}+1\right)=2 N+n .
$$

Now by (4.31) of Theorem 4.3,

$$
i(x, 1)=i\left(x, 2 m_{j}+1\right)-2 N=n .
$$

Remark 5.1. Although the global minimal point $(\tau, x)$ of $f$ on $E$ satisfies $i_{\tau}(x)=n$, it is not clear whether $[(\tau, x)] \in \mathcal{V}_{\infty}(\Sigma, \alpha)$.

Now we come to the proof of Theorem 1.2. We will prove the following stronger version. For every $(\tau, x) \in \mathcal{J}(\Sigma, \alpha)$, the elliptic height $e(x)$ of $(\tau, x)$ is defined to be the elliptic height $e\left(\gamma_{x}(\tau)\right)$, where $\gamma_{x}$ is the associated symplectic path of $(\tau, x)$.

Theorem 5.2. Let $\Sigma \in \mathcal{H}(2 n)$ and $\alpha \in(1,2)$. Suppose $\# \tilde{\mathcal{J}}(\Sigma)<+\infty$. By the notation introduced in the proof of Theorem 5.1, and specifically, the proof of Claim 1 in the proof of Theorem 5.1 , for each $s=1, \ldots, \varrho_{n}(\Sigma)$, there exists a unique $j(s) \in\{1, \ldots, q\}$ such that $p(N-s+1)=\left(\left[\left(\tau_{j(s)}, x_{j(s)}\right)\right], 2 m_{j(s)}\right)$. Then

$$
e\left(x_{j(s)}\right) \geq 2|n-2 s+1|+2, \quad \text { for all } s=1, \ldots, \varrho_{n}(\Sigma) .
$$

Specially, $\left[\left(\tau_{j(1)}, x_{j(1)}\right)\right]$ is an elliptic element in $\mathcal{V}_{\infty}(\Sigma, \alpha)$.

Proof. By the definition of the injection map $p$ in Lemma 3.1, we have

$$
i\left(x_{j(s)}, 2 m_{j(s)}\right) \leq 2 N-2 s+n \leq i\left(x_{j(s)}, 2 m_{j(s)}\right)+\nu\left(x_{j(s)}, 2 m_{j(s)}\right)-1 .
$$

By (2.41) of Theorem 2.3 and (4.31) in Theorem 4.3,

$$
\begin{aligned}
\frac{e\left(\tau_{j(s)}, x_{j(s)}\right)}{2} \geq & i\left(x_{j(s)}, 2 m_{j(s)}\right)+\nu\left(x_{j(s)}, 2 m_{j(s)}\right) \\
& -i\left(x_{j(s)}, 2 m_{j(s)}+1\right)+i\left(x_{j(s)}, 1\right)+1 \\
= & i\left(x_{j(s)}, 2 m_{j(s)}\right)+\nu\left(x_{j(s)}, 2 m_{j(s)}\right)-2 N+1 \\
\geq & 2 N-2 s+n+1-2 N+1 \\
= & n-2 s+2
\end{aligned}
$$

where (5.24) follows from the right-hand inequality in (5.22). 
On the other hand, by (2.42) in Theorem 2.3,

$$
\begin{aligned}
\frac{e\left(\tau_{j(s)}, x_{j(s)}\right)}{2} \geq & i\left(x_{j(s)}, 2 m_{j(s)}+1\right)+\nu\left(x_{j(s)}, 2 m_{j(s)}+1\right) \\
& -i\left(x_{j(s)}, 2 m_{j(s)}\right)-i\left(x_{j(s)}, 1\right)-\nu\left(x_{j(s)}, 1\right) .
\end{aligned}
$$

By (4.29) and (4.31) of Theorem 4.3 and the left-hand inequality in (5.22),

$$
\begin{aligned}
\frac{e\left(\tau_{j(s)}, x_{j(s)}\right)}{2} & \geq 2 N-(2 N-2 s+n) \\
& =2 s-n .
\end{aligned}
$$

Now (5.24) and (5.26) yield (5.21).

The following theorem implies Theorem 1.3. Note that by Theorem 5.1 $\varrho_{n}(\Sigma)-1 \geq\left[\frac{n}{2}\right]$ always.

Theorem 5.3. Let $\Sigma \in \mathcal{H}(2 n)$ with $n \geq 2$ and $\alpha \in(1,2)$. Suppose $\# \tilde{\mathcal{J}}(\Sigma)<+\infty$. Then there exist at least $\varrho_{n}(\Sigma)-1$ elements in $\mathcal{V}_{\infty}(\Sigma, \alpha)$ such that each such an element $[(\tau, x)]$ satisfies

$$
\hat{i}(x, 1) \in \mathbf{R} \backslash \mathbf{Q} .
$$

Specifically, such a closed characteristic $[(\tau, x)]$ must possess at least a rotator on $\mathbf{U}$ in the sense of Theorem 2.1 with an irrational multiple of $2 \pi$ rotation angle.

Proof. Since $n \geq 2$, we have $\varrho_{n}(\Sigma)-1 \geq 1$. Let

$$
\mathcal{V}_{\infty}(\Sigma, \alpha)=\left\{\left[\left(\tau_{j}, x_{j}\right)\right] \mid 1 \leq j \leq q\right\} .
$$

By (4.39) and Corollary 2.1, the $D_{j}$ defined by (4.2) according to Step 1 of the proof of Theorem 4.3 satisfies

$$
D_{j}=\hat{i}\left(x_{j}, 1\right), \quad \text { for all } j=1, \ldots, q .
$$

By Step 1 of the proof of Theorem 5.1, we obtain $\left(N, m_{1}, \ldots, m_{q}\right) \in \mathbf{N}^{q+1}$ such that the injection map $p$ of Lemma 3.1 satisfies (5.14). Reordering elements in $\mathcal{V}_{\infty}(\Sigma, \alpha)$ to simplify notation, we can assume

$$
p(N-j+1)=\left(\left[\tau_{j}, x_{j}\right], 2 m_{j}\right), \quad \text { for all } j=1, \ldots, \varrho_{n}(\Sigma) .
$$

Thus by Lemma 3.1 and (1.28),

$$
\begin{aligned}
2 m_{k} D_{k}-2 m_{j} D_{j} & =2 m_{k} \hat{i}\left(x_{k}, 1\right)-2 m_{j} \hat{i}\left(x_{j}, 1\right) \\
& =\hat{i}\left(x_{k}, 2 m_{k}\right)-\hat{i}\left(x_{j}, 2 m_{j}\right) \\
& <0, \quad \text { if } 1 \leq j<k \leq \varrho_{n}(\Sigma) .
\end{aligned}
$$

Now it suffices to prove that among the first $\varrho_{n}(\Sigma)$ of $D_{j}$ 's, at most one of them is rational. We prove this claim indirectly by assuming

$$
D_{j} \text { and } D_{k} \in \mathbf{Q} \text {, }
$$


for some $j$ and $k$ satisfying $1 \leq j<k \leq \varrho_{n}(\Sigma)$. Then in our choice of $\left(N, m_{1}, \ldots, m_{q}\right)$ in the proof of Theorem 4.1, we require that $N \in \mathbf{N}$ further satisfies

$$
\frac{N}{M D_{i}} \in \mathbf{N}, \quad \text { for } i=j \text {, and } k \text {. }
$$

Then the closure of the set $\{\{N v\} \mid N \in \mathbf{N}$ satisfies (5.32) $\}$ is still a closed additive subgroup of $\mathbf{T}^{h}$ for some $h \in \mathbf{N}$. Thus (4.22) holds in the $j^{\text {th }}$ and $k^{\text {th }}$ coordinates for infinitely many $N$, if we choose $\chi$ to be a vertex of $[0,1]^{h}$ with $\chi_{j}=\chi_{k}=0$. This implies

$$
\left|\left\{\frac{N}{M D_{i}}\right\}\right|=0, \quad \text { for } i=j, k .
$$

Therefore by the definition (4.10) of $m_{i}$ 's,

$$
m_{i}=\left[\frac{N}{M D_{i}}\right] M=\frac{N}{D_{i}}, \quad \text { for } i=j, k
$$

This yields

$$
m_{j} D_{j}=N=m_{k} D_{k},
$$

contradicts (5.30), and completes the proof of the theorem.

The following theorem shows the existence of multiple elliptic orbits and implies Theorem 1.4.

Theorem 5.4. Let $\Sigma \in \mathcal{H}(2 n)$ with $n \geq 2$ and $\alpha \in(1,2)$. Suppose $\# \tilde{\mathcal{J}}(\Sigma)<+\infty$ and

$$
{ }^{\#} \mathcal{V}_{\infty}(\Sigma, \alpha) \leq 2 \varrho_{n}(\Sigma)-2
$$

Then there exist at least two elliptic elements in $\mathcal{V}_{\infty}(\Sigma, \alpha)$.

In particular, by (1.8), there are at least two elliptic elements in $\mathcal{V}_{\infty}(\Sigma, \alpha)$, provided

$$
{ }^{\#} \mathcal{V}(\Sigma, \alpha) \leq 2\left[\frac{n}{2}\right]
$$

Proof. Let $\mathcal{V}_{\infty}(\Sigma, \alpha)=\left\{\left[\left(\tau_{j}, x_{j}\right)\right] \mid 1 \leq j \leq q\right\}$. Let $v \in \mathbf{R}^{k}$ be the vector given by (4.21) according to quantities in (4.40)-(4.41) in the proof of Theorem 4.3. Let $A(v)$ be the set given by Theorem 4.2 according to this $v$.

We use notation introduced in the proof of Theorem 5.3. Note that by Theorem 5.1 and the fact that $n \geq 2, q \geq \varrho_{n}(\Sigma) \geq 2$. By Theorem 5.3, $D_{j} \in$ $\mathbf{R} \backslash \mathbf{Q}$ holds for at least $[n / 2]$ integers in $\{1, \ldots, q\}$. Therefore by Theorem 4.2,

$$
\operatorname{dim} A(v) \geq 1, \quad 0 \notin A(v) .
$$

We continue our proof in two steps. 
Step 1. For a given $a \in A(v)$, we define $\chi \equiv \chi(a)=\left(\psi\left(a_{1}\right), \ldots, \psi\left(a_{k}\right)\right)$ by (4.26). Let $\left(N, m_{1}, \ldots, m_{q}\right) \in \mathbf{N}^{q+1}$ be given in Step 1 of the proof for Theorem 5.1 via an application of Theorems 4.1 to 4.3 with this $\chi=\chi(a)$ used in their proofs. By the proof of Theorem 5.1, there exists a subset $L(a, N)$ of $\{1, \ldots, q\}$ such that

(i) $1 \in L(a, N)$ and ${ }^{\#} L(a, N)=\varrho_{n}(\Sigma)$,

(ii) $p(N)=\left(\left[\left(\tau_{1}, x_{1}\right), m_{1}\right)\right]$ and $x_{1}$ is elliptic;

(iii) For each $s=2, \ldots, \varrho_{n}(\Sigma)$, there is a $j(s) \in L(a, N) \backslash\{1\}$ such that

$$
p(N-s+1)=\left(\left[\tau_{j(s)}, x_{j(s)}\right], m_{j(s)}\right) .
$$

By Lemma 3.1, we have

$$
m_{1} D_{1}-m_{j} D_{j}>0, \quad \text { for all } j \in L(a, N) \backslash\{1\} .
$$

Assuming that there exists only one elliptic element in $\mathcal{V}_{\infty}(\Sigma, \alpha)$, we always get the same elliptic element $\left[\left(\tau_{1}, x_{1}\right)\right] \in \mathcal{V}_{\infty}(\Sigma, \alpha)$ for all $a \in A(v)$.

By (5.36), we have $-a \in A(v)$. By definition (4.26), we have $\chi(-a) \neq$ $\chi(a)$. Thus by the proof of Theorem 5.1 , for this vertex $\chi(-a)$ we get $\left(\tilde{N}, \tilde{m}_{1}, \ldots, \tilde{m}_{q}\right)$ $\in \mathbf{N}^{q+1}$ and another subset $L(-a, \tilde{N})$ of $\{1, \ldots, q\}$ such that Theorems 5.1-5.3 hold, specially the above (i)-(iii) still hold correspondingly.

By the assumption $q \leq 2 \varrho_{n}(\Sigma)-2$ of (5.34),

$$
1 \leq \#((L(a, N) \cap L(-a, \tilde{N})) \backslash\{1\}) \leq \varrho_{n}(\Sigma) .
$$

Let

$$
t_{0}=\frac{\delta_{1}}{6(|a|+1)(M \Lambda+1)}, \quad \Lambda=\max _{1 \leq j \leq q} D_{j} .
$$

Note that in the proof of Theorem 4.1 we can further require $N \in \mathbf{N}$ such that the vector $\{N v\}-\chi(a)$ defined by (4.22) and (4.21) are located in a sufficiently small neighborhood inside the open ball in $V$ with radius $\delta_{1} /(6 M \Lambda+1)$ and centered at $a t_{0}$; i.e.,

$$
\begin{aligned}
& \{N v\}-\chi(a) \in V, \\
& \left|\{N v\}-\chi(a)-a t_{0}\right|<\frac{\delta_{1}}{6 M \Lambda+1},
\end{aligned}
$$

where $V=T_{0} \pi^{-1}(\overline{\{\{N v\} \mid N \in \mathbf{N}\}})$ is as defined in Theorem 4.2. Under this requirement, we still have (4.22):

$$
\begin{aligned}
\left|\left\{\frac{N}{M D_{i}}\right\}-\chi_{i}\right| & <\left|a_{i} t_{0}\right|+\frac{\delta_{1}}{6 M \Lambda+1} \leq \frac{\delta_{1}}{3 M \Lambda}, \\
\left|\left\{\frac{N \alpha_{i, j}}{D_{i}}\right\}-\chi_{i, j}\right| & <\left|a_{i, j} t_{0}\right|+\frac{\delta_{1}}{6 M \Lambda+1} \leq \frac{\delta_{1}}{3}
\end{aligned}
$$


for infinitely many $N \in \mathbf{N}$. Here $\chi_{i}=\psi\left(a_{i}\right)$ and $\chi_{i, j}=\psi\left(a_{i, j}\right)$; the function $\psi$ is defined in Theorem 4.2.

Claim. $a_{1} D_{1}-a_{j} D_{j}=0$ for all $j \in(L(a, N) \cap L(-a, \tilde{N})) \backslash\{1\}$.

Assuming the claim does not hold, we prove it by contradiction. In fact, we can further require $N \in \mathbf{N}$ so that the following also holds:

$\left|\left\{\frac{N}{M D_{k}}\right\}-\chi_{k}-a_{k} t_{0}\right|<\frac{t_{0}}{3 \Lambda} \min _{a_{i} D_{i}-a_{j} D_{j} \neq 0}\left|a_{i} D_{i}-a_{j} D_{j}\right|, \quad$ for all $1 \leq k \leq q$.

By our choice of $\left(N, m_{1}, \ldots, m_{q}\right)$, we have

$$
\begin{aligned}
m_{1} D_{1}-m_{j} D_{j}= & M\left(\left[\frac{N}{M D_{1}}\right]+\chi_{1}\right) D_{1}-M\left(\left[\frac{N}{M D_{j}}\right]+\chi_{j}\right) D_{j} \\
= & M\left(\left(\chi_{1}-\left\{\frac{N}{M D_{1}}\right\}\right) D_{1}-\left(\chi_{j}-\left\{\frac{N}{M D_{j}}\right\}\right) D_{j}\right) \\
= & -M t_{0}\left(a_{1} D_{1}-a_{j} D_{j}\right)+M\left(\left(\chi_{1}-\left\{\frac{N}{M D_{1}}\right\}+a_{1} t_{0}\right) D_{1}\right. \\
& \left.-\left(\chi_{j}-\left\{\frac{N}{M D_{j}}\right\}+a_{j} t_{0}\right) D_{j}\right) .
\end{aligned}
$$

For any $j \in(L(a, N) \cap L(-a, \tilde{N})) \backslash\{1\}$, by (5.37) and (5.45),

$$
a_{1} D_{1} \leq a_{j} D_{j}
$$

For any $j>1$ satisfying $j \in L(a, N) \cap L(-a, \tilde{N})$, we apply the above argument to $a$ and $-a$ respectively and obtain $a_{1} D_{1} \leq a_{j} D_{j}$ and $-a_{1} D_{1} \leq$ $-a_{j} D_{j}$. Therefore $a_{1} D_{1}=a_{j} D_{j}$, and the claim is proved.

Step 2. Set

$$
\begin{aligned}
V_{j} & =\left\{a \in V \mid a_{1} D_{1}=a_{j} D_{j}\right\}, \quad j=2, \ldots, q, \\
B(v) & =A(v) \backslash \bigcup_{V_{j} \neq V, j=2, \ldots, q} V_{j} .
\end{aligned}
$$

Since $\operatorname{dim} A(v) \geq 1$, by Theorem 4.2,A(v) is obtained from $V$ by deleting finitely many proper linear subspaces of $V$, and so is $B(v)$. Hence $B(v)$ is nonempty.

Now we choose an $a \in B(v)$ and $j \in L(a, N) \cap L(-a, \tilde{N}) \backslash\{1\}$. By the Claim in Step 1, we have $a_{1} D_{1}=a_{j} D_{j}$. By definitions of $a \in B(v)$ and $j$ we have $V_{j}=V$.

By (5.40), the vector $\{N v\}-\chi$ defined by (4.22) belongs to $V$, and thus belongs to $V_{j}$. Then by definition (5.47) of $V_{j}$, this implies

$$
0=\left(\left\{N v_{1}\right\}-\chi_{1}\right) D_{1}-\left(\left\{N v_{j}\right\}-\chi_{j}\right) D_{j} .
$$


By definition (4.21) of $v$,

$$
0=\left(\left\{\frac{N}{M D_{1}}\right\}-\chi_{1}\right) D_{1}-\left(\left\{\frac{N}{M D_{j}}\right\}-\chi_{j}\right) D_{j} .
$$

By (5.44), this implies $m_{1} D_{1}=m_{j} D_{j}$. Then it contradicts (5.37) and completes the proof.

\section{Appendix 1. The Maslov-type index and its iteration theory}

In this section we give a brief review of the Maslov-type index and its iteration theory for symplectic matrix paths. We use notation already introduced in Section 1.

Definition 6.1 (cf. [23] and [29]). For $\tau>0$ and $\omega \in \mathbf{U}$, given two paths $\gamma_{0}$ and $\gamma_{1} \in \mathcal{P}_{\tau}(2 n)$, if there exists a map $\delta \in C([0,1] \times[0, \tau], \operatorname{Sp}(2 n))$ such that $\delta(0, \cdot)=\gamma_{0}(\cdot), \delta(1, \cdot)=\gamma_{1}(\cdot), \delta(s, 0)=I$, and $\nu_{\omega}(\delta(s,)$.$) is constant for$ $0 \leq s \leq 1$, then $\gamma_{0}$ and $\gamma_{1}$ are $\omega$-homotopic on $[0, \tau]$ along $\delta(\cdot, \tau)$ and $\gamma_{0} \sim_{\omega} \gamma_{1}$. If $\gamma_{0} \sim \omega \gamma_{1}$ on $[0, \tau]$ along $\delta(\cdot, \tau)$ for all $\omega \in \mathbf{U}$, then $\gamma_{0}$ and $\gamma_{1}$ are homotopic on $[0, \tau]$ along $\delta(\cdot, \tau)$ and we write $\gamma_{0} \sim \gamma_{1}$.

We define $2 \times 2$ and $4 \times 4$ matrices

$$
\begin{array}{rlrl}
D(a) & =\left(\begin{array}{cc}
a & 0 \\
0 & \frac{1}{a}
\end{array}\right), & & R(\theta)=\left(\begin{array}{cc}
\cos \theta & -\sin \theta \\
\sin \theta & \cos \theta
\end{array}\right), \\
N_{1}(\lambda, c) & =\left(\begin{array}{cc}
\lambda & c \\
0 & \lambda
\end{array}\right), & N_{2}(\omega, b)=\left(\begin{array}{cc}
R(\theta) & b \\
0 & R(\theta)
\end{array}\right),
\end{array}
$$

for $a, \lambda, c, \theta \in \mathbf{R}, \omega \in \mathbf{U}$, and $b \in \mathcal{L}\left(\mathbf{R}^{2}\right)$. They are called basic normal forms of symplectic matrices in [29] when $a= \pm 2, \theta \in \mathbf{R}, \lambda= \pm 1, c \in \mathbf{R}$, $\omega=e^{\theta \sqrt{-1}} \in \mathbf{U} \backslash \mathbf{R}$ with $\theta \in \mathbf{R} ; b=\left(\begin{array}{ll}b_{1} & b_{2} \\ b_{3} & b_{4}\end{array}\right)$ is a $2 \times 2$ real matrix with $b_{2}-b_{3} \neq 0$. In [29], a basic normal form matrix $M \in \operatorname{Sp}(2 n)$ is called trivial if $\sigma\left(M R((t-1) \varepsilon)^{\diamond n}\right) \cap \mathbf{U}=\emptyset$ for $\varepsilon>0$ small enough and $t \in[0,1)$. Note that the normal forms $N_{1}(1,-1), N_{1}(-1,1), D( \pm 2), N_{2}(\omega, b)$ and $N_{2}(\bar{\omega}, b) \in \mathcal{M}_{\omega}^{1}(4)$ with $\omega=\exp (\theta \sqrt{-1}) \in \mathbf{U} \backslash \mathbf{R}$ and $\left(b_{2}-b_{3}\right) \sin \theta>0$ are trivial, and all other basic normal form matrices are nontrivial. Here using Definition 1.2 we define

$$
\mathcal{M}_{\omega}^{1}(2 n)=\left\{M \in \operatorname{Sp}(2 n) \mid \nu_{\omega}(M)=1\right\} .
$$

For any $\tau>0, n \in \mathbf{N}$, and $k \in \mathbf{Z}$, following [23], we define

$$
\begin{aligned}
\phi_{\theta, \tau}(t) & =R\left(\frac{\theta t}{\tau}\right), \quad \text { for all } t \in[0, \tau], \\
\chi_{\tau}(t) & =D\left(1+\frac{t}{\tau}\right), \quad \text { for all } t \in[0, \tau] ;
\end{aligned}
$$

We also denote by $N_{1}\left(e^{\theta \sqrt{-1}}, b\right)=R(\theta)$ for any $b \in \mathbf{R}$ and $\theta \in \mathbf{R} \backslash(\pi \mathbf{Z})$. 
The following theorem gives a characterization of our index function theory.

Theorem 6.1 (Theorem 2.11 of [29]). For any $\tau>0$ and $\omega=\exp (\theta \sqrt{-1})$ $\in \mathbf{U}$, there exists a unique function $i_{\omega}: \cup_{n \in \mathbf{N}} \mathcal{P}_{\tau}(2 n) \rightarrow \mathbf{Z}$ satisfying the following five axioms:

$1^{\circ}$ (Homotopy invariant). For $\gamma_{0}$ and $\gamma_{1} \in \mathcal{P}_{\tau}(2 n)$, if $\gamma_{0} \sim_{\omega} \gamma_{1}$ on $[0, \tau]$, then

$$
i_{\omega}\left(\gamma_{0}\right)=i_{\omega}\left(\gamma_{1}\right)
$$

$2^{\circ}$ (Symplectic additivity). For any $\gamma_{i} \in \mathcal{P}_{\tau}\left(2 n_{i}\right)$ with $i=0$ and 1 ,

$$
i_{\omega}\left(\gamma_{0} \diamond \gamma_{1}\right)=i_{\omega}\left(\gamma_{0}\right)+i_{\omega}\left(\gamma_{1}\right)
$$

$3^{\circ}$ (Clockwise continuity). For any $\gamma \in \mathcal{P}_{\tau}(2)$ and $\omega \in \mathbf{U}$ satisfying $\gamma(\tau)=N_{1}(\omega, b)$, there exists a $\theta_{0}>0$ such that

$$
i_{\omega}\left(\left(\gamma(\tau) \phi_{-\theta, \tau}\right) * \gamma\right)=i_{\omega}(\gamma), \quad \text { for all } 0<\theta \leq \theta_{0} .
$$

$4^{\circ}$ (Counterclockwise jumping). For any $\gamma \in \mathcal{P}_{\tau}(2)$ and $\omega \in \mathbf{U}$ satisfying $\gamma(\tau)=N_{1}(\omega, b)$, there exists a $\theta_{0}>0$ such that

$$
i_{\omega}\left(\left(\gamma(\tau) \phi_{\theta, \tau}\right) * \gamma\right)=i_{\omega}(\gamma)+1, \quad \text { for all } 0<\theta \leq \theta_{0} .
$$

$5^{\circ}$ (Normality).

$$
i_{\omega}\left(\chi_{\tau}\right)=0
$$

Theorem 6.2 (Theorem 2.13 of [29]). For any two paths $\gamma_{0}$ and $\gamma_{1} \in \mathcal{P}_{\tau}(2 n)$ with $i_{\omega}\left(\gamma_{0}\right)=i_{\omega}\left(\gamma_{1}\right)$, suppose that there exists a continuous path $h:[0,1] \rightarrow \operatorname{Sp}(2 n)$ such that $h(0)=\gamma_{0}(\tau), h(1)=\gamma_{1}(\tau)$, and $\operatorname{dim}_{\mathbf{C}} \operatorname{ker}_{\mathbf{C}}(h(s)-\omega I)=\nu_{\tau, \omega}\left(\gamma_{0}\right)$ for all $s \in[0,1]$. Then $\gamma_{0} \sim_{\omega} \gamma_{1}$ on $[0, \tau]$ along $h$.

Theorem 6.3 (Theorem 1.4 of [29]). For any $\tau>0, \gamma \in \mathcal{P}_{\tau}(2 n)$, and $k \in \mathbf{N}$,

$$
i(\gamma, k)=\sum_{\omega^{k}=1} i_{\omega}(\gamma), \quad \nu(\gamma, k)=\sum_{\omega^{k}=1} \nu_{\omega}(\gamma)
$$

Theorem 6.4 (Theorem 1.5 of [29]). For any $\tau>0$ and $\gamma \in \mathcal{P}_{\tau}(2 n)$,

$$
\hat{i}(\gamma, 1) \equiv \lim _{k \rightarrow+\infty} \frac{i(\gamma, k)}{k}=\frac{1}{2 \pi} \int_{\mathbf{U}} i_{\omega}(\gamma) d \omega
$$

Specially, $\hat{i}(\gamma, 1)$ is always a finite real number, which is called the mean index per $\tau$ of $\gamma$. 
Definition 6.2 (Definition 1.1 of [29]). For any $M \in \operatorname{Sp}(2 n)$, define the homotopy set of $M$ in $\operatorname{Sp}(2 n)$ by

$$
\begin{aligned}
\Omega(M)= & \{N \in \operatorname{Sp}(2 n) \mid \sigma(N) \cap \mathbf{U}=\sigma(M) \cap \mathbf{U}, \text { and } \\
& \left.\operatorname{dim}_{\mathbf{C}} \operatorname{ker}_{\mathbf{C}}(N-\lambda I)=\operatorname{dim}_{\mathbf{C}} \operatorname{ker}_{\mathbf{C}}(M-\lambda I), \text { for all } \lambda \in \sigma(M) \cap \mathbf{U}\right\} .
\end{aligned}
$$

We denote by $\Omega^{0}(M)$ the path connected component of $\Omega(M)$ which contains $M$, and call it the homotopy component of $M$ in $\operatorname{Sp}(2 n)$. Also, $N \approx M$ if $N \in \Omega^{0}(M)$. This is an equivalent relation in $\operatorname{Sp}(2 n)$. The equivalent class of $M$ in $\operatorname{Sp}(2 n)$ is called the homotopy type of $M$.

Theorem 6.5 (Theorem 7.8 of [29]). For any $M \in \operatorname{Sp}(2 n)$, we have

$$
\begin{aligned}
M \approx & N_{1}(1,1)^{\diamond p_{-}} \diamond I_{2 p_{0}} \diamond N_{1}(1,-1)^{\diamond p_{+}} \diamond N_{1}(-1,1)^{\diamond q_{-}} \diamond\left(-I_{2 q_{0}}\right) \\
& \diamond N_{1}(-1,-1)^{\diamond q_{+}} \diamond R\left(\theta_{1}\right) \diamond \cdots \diamond R\left(\theta_{r}\right) \diamond N_{2}\left(\omega_{1}, u_{1}\right) \diamond \cdots \diamond N_{2}\left(\omega_{r_{*}}, u_{r_{*}}\right) \\
& \diamond N_{2}\left(\lambda_{1}, v_{1}\right) \diamond \cdots \diamond N_{2}\left(\lambda_{r_{0}}, v_{r_{0}}\right) \diamond M_{k},
\end{aligned}
$$

where $p_{-}, p_{0}, p_{+}, q_{-}, q_{0}, q_{+}, r, r_{*}, r_{0}$, and $k$ are nonnegative integers; $\omega_{j}=$ $e^{\sqrt{-1} \alpha_{j}}, \lambda_{j}=e^{\sqrt{-1} \beta_{j}}$; the real numbers $\theta_{j}$ with $1 \leq j \leq r, \alpha_{h}$ with $1 \leq h \leq r_{*}$, $\beta_{l}$ with $1 \leq l \leq r_{0}$, are in $(0, \pi) \cup(\pi, 2 \pi)$ provided the corresponding $r, r_{*}$, or $r_{0}>0$ respectively; $N_{2}\left(\omega_{j}, u_{j}\right)$ 's are nontrivial and $N_{2}\left(\lambda_{j}, v_{j}\right)$ 's are trivial basic normal forms; $M_{k}=D(2)^{\diamond k}$ or $D(-2) \diamond D(2)^{\diamond(k-1)}$. All these integers, real numbers, and basic normal form matrices are uniquely determined by $M$. It holds that

$$
p_{-}+p_{0}+p_{+}+q_{-}+q_{0}+q_{-}+r+2 r_{*}+2 r_{0}+k=n .
$$

Definition 6.3 (Definition 4.8 of [29]). For any basic normal form $M \in$ $\operatorname{Sp}(2 n)$ and $\omega \in \mathbf{U} \cap \sigma(M)$, we define the ultimate type $(p, q)$ of $\omega$ for $M$ to be its usual Krein type if $M$ is nontrivial, and to be $(0,0)$ if $M$ is trivial. When $\omega \in \mathbf{U} \backslash \sigma(M)$ with $M \in \operatorname{Sp}(2 n)$, we define the ultimate type of $\omega$ for $M$ to be $(0,0)$. For any $M \in \operatorname{Sp}(2 n)$, by Theorem 6.5 there exists a $\diamond$-product expansion (6.3) in the homotopy component $\Omega^{0}(M)$ of $M$ as:

$$
M \approx M_{1} \diamond M_{2} \diamond \cdots \diamond M_{k} \diamond M_{0},
$$

where each $M_{i}$ is a basic normal form for $1 \leq i \leq k$ and $\sigma\left(M_{0}\right) \cap \mathbf{U}=\emptyset$. Denote the ultimate type of $\omega$ for $M_{i}$ by $\left(p_{i}, q_{i}\right)$ for $0 \leq i \leq k$. Let $p=\sum_{i=0}^{k} p_{i}$ and $q=\sum_{i=0}^{k} q_{i}$. We define the ultimate type of $\omega$ for $M$ by $(p, q)$.

The following theorem characterizes the splitting numbers algebraically.

Theorem 6.6 (Lemma 4.5 and Theorem 4.11 of [29]). For any $\omega \in \mathbf{U}$ and $M \in \operatorname{Sp}(2 n)$, both $S_{N}^{+}(\omega)$ and $S_{N}^{+}(\omega)$ are constants for any $N \in \Omega^{0}(M)$, and

$$
S_{M}^{+}(\omega)=p \quad \text { and } \quad S_{M}^{-}(\omega)=q
$$

where $(p, q)$ is the ultimate type of $\omega$ for $M$. 
Theorem 6.7 (Theorem 1.3 of [31]). For $\tau>0$, let $\gamma \in \mathcal{P}_{\tau}(2 n)$. In Theorem 6.5 we let $M=\gamma(\tau)$ and use the notation there. Then for any $m \in \mathbf{N}$,

$$
\begin{aligned}
i(\gamma, m)= & m\left(i(\gamma, 1)+p_{-}+p_{0}-r\right)+2 \sum_{j=1}^{r} E\left(\frac{m \theta_{j}}{2 \pi}\right)-p_{-} \\
& -p_{0}-\frac{1+(-1)^{m}}{2}\left(q_{0}+q_{+}\right)-r+2\left(\sum_{j=1}^{r_{*}} \phi\left(\frac{m \alpha_{j}}{2 \pi}\right)-r_{*}\right), \\
\nu(\gamma, m)= & \nu(\gamma, 1)+\frac{1+(-1)^{m}}{2}\left(q_{-}+2 q_{0}+q_{+}\right)+2 \varphi(m, \gamma(\tau)),
\end{aligned}
$$

where

$$
\begin{aligned}
\varphi(m, \gamma(\tau))= & \left(r-\sum_{j=1}^{r} \phi\left(\frac{m \theta_{j}}{2 \pi}\right)\right)+\left(r_{*}-\sum_{j=1}^{r_{*}} \phi\left(\frac{m \alpha_{j}}{2 \pi}\right)\right) \\
& +\left(r_{0}-\sum_{j=1}^{r_{0}} \phi\left(\frac{m \beta_{j}}{2 \pi}\right)\right) .
\end{aligned}
$$

Here the functions $E(\cdot)$ and $\phi(\cdot)$ are as defined at the beginning of Section 2.

\section{Appendix 2. The Fadell-Rabinowitz $S^{1}$-cohomological index. (By John Mather)}

For a principal $U(1)$-bundle $E \rightarrow B$, the Fadell-Rabinowitz index of $E$ is defined to be

$$
\sup \left\{k \mid c_{1}(E)^{k-1} \neq 0\right\},
$$

where $c_{1}(E) \in H^{2}(B, \mathbf{Q})$ is the first rational Chern class. For a $U(1)$-space, i.e., a topological space $X$ with a $U(1)$-action, the Fadell-Rabinowitz index is defined to be the index of the bundle

$$
X \times S^{\infty} \rightarrow X \times_{U(1)} S^{\infty},
$$

where $S^{\infty} \rightarrow C P^{\infty}$ is the universal $U(1)$-bundle.

Acknowledgements. The authors sincerely thank Professors Helmut Hofer, John Mather, and the referee for their careful reading and many valuable comments on this paper, which make it more readable, with special thanks to Professor John Mather for Appendix 2, and to the referee for pointing out the equivalent definition (1.19) of the index function in the degenerate case. 
Nankai Institute of Mathematics, Nankai University, Tianjin 300071, The People's Republic of China

E-mail addresses: longym@nankai.edu.cn, zhucf@nankai.edu.cn

REFERENCES

[1] A. Ambrosetti and G. Mancini, On a theorem by Ekeland and Lasry concerning the number of periodic Hamiltonian trajectories, J. Differential Equations 43 (1981), 1-6.

[2] V. I. ARnol'D, On a characteristic class entering into conditions of quantization, Funkt. Anal. i Priložen 1 (1967), 1-14 (Russian); Funct. Anal. Appl. 1 (1967), 1-13 (English transl.).

[3] T. Bartsch, A generalization of the Weinstein-Moser theorems on periodic orbits of a Hamiltonian system near an equilibrium, Ann. Inst. H. Poincaré Anal. Non Linéaire 14 (1997), 691-718.

[4] H. Berestycki, J. M. Lasry, G. Mancini, and B. Ruf, Existence of multiple periodic orbits on star-shaped Hamiltonian surfaces, Comm. Pure Appl. Math. 38 (1985), 253-290.

[5] R. Вотт, On the iteration of closed geodesics and the Sturm intersection theory, Comm. Pure Appl. Math. 9 (1956), 171-206.

[6] V. Brousseau, L'index d'un système hamiltonien linéaire, C. R. Acad. Sci. Paris Ser. I Math. 303 (1986), 351-354.

[7] C. Conley and E. Zehnder, Morse-type index theory for flows and periodic solutions for Hamiltonian equations, Comm. Pure Appl. Math. 37 (1984), 207-253.

[8] G. Dell'Antonio, B. D'Onofrio, and I. Ekeland, Les systèmes hamiltoniens convexes et pairs ne sont pas ergodiques en general, C. R. Acad. Sci. Paris Ser. I Math. 315 (1992), $1413-1415$.

[9] D. Dong and Y. Long, The iteration formula of the Maslov-type index theory with applications to nonlinear Hamiltonian systems, Trans. A. M. S. 349 (1997), 2619-2661.

[10] I. Ekeland, Une théorie de Morse pour les systèmes hamiltoniens convexes, Ann. Inst. H. Poincaré Anal. Non Linéaire 1 (1984), 19-78.

[11] An index theory for periodic solutions of convex Hamiltonian systems, in Nonlinear Funct. Anal. and its Appl., Proc. Symposia in Pure Math. 45 (1986), 395-423.

[12] Convexity Methods in Hamiltonian Mechanics, Springer-Verlag, New York (1990).

[13] I. Ekeland and H. Hofer, Convex Hamiltonian energy surfaces and their periodic trajectories, Comm. Math. Phys. 113 (1987), 419-467.

[14] I. Ekeland and J.-M. Lasry, On the number of periodic trajectories for a Hamiltonian flow on a convex energy surface, Ann. of Math. 112 (1980), 283-319.

[15] I. Ekeland and L. Lassoued, Multiplicité des trajectories fermées d'un systémes hamiltoniens sur une hypersurface d'énergie convexes, Ann. Inst. H. Poincaré Anal. Non Linéaire 4 (1987), 1-29.

[16] E. Fadell and P. Rabinowitz, Generalized cohomological index theories for Lie group actions with an application to bifurcation questions for Hamiltonian systems, Invent. Math. 45 (1978), 139-174.

[17] M. Girardi, Multiple orbits for Hamiltonian systems on starshaped energy surfaces with symmetry, Ann. Inst. H. Poincaré Anal. Non Linéaire 1 (1984), 285-294.

[18] J. Han and Y. Long, Normal forms of symplectic matrices (II), Acta Sci. Natur. Univ. Nankaiensis 32 (1999), 30-41.

[19] H. Hofer, A new proof for a result of Ekeland and Lasry concerning the number of periodic Hamiltonian trajectories on a prescribed energy surfaces, Boll. Un. Mat. Ital. 1 (1982), 931-942.

[20] H. Hofer, K. Wysocki, and E. Zehnder, The dynamics on three-dimensional strictly convex energy surfaces, Ann. of Math. 148 (1998), 197-289. 
[21] V. J. Horn, Beiträge zur Theorie der kleinen Schwingungen, Zeit. Math. Phys. 48 (1903), 400-434.

[22] A. Liapunov, Problème général de la stabilité du mouvement, Russian edition (1892), Ann. Fac. Sci. Toulouse 9 (1907), 203-474.

[23] Y. Long, Maslov-type index, degenerate critical points, and asymptotically linear Hamiltonian systems, Science in China Ser. A (Scientia Sinica) 7 (1990), 673-682 (Chinese edition); 33 (1990), 1409-1419. (English edition).

[24] The structure of the singular symplectic matrix set, Science in China (Scientia Sinica), Ser. A 5 (1991), 457-465 (Chinese edition); 34 (1991), 897-907 (English edition).

[25] _ The Index Theory of Hamiltonian Systems with Applications (in Chinese), Science Press, Beijing (1993).

[26] A Maslov-type index theory for symplectic paths, Topol. Methods Nonlinear Anal. 10 (1997), 47-78.

[27] Hyperbolic closed characteristics on compact convex smooth hypersurfaces in $\mathbf{R}^{2 n}$, J. Differential Equations 150 (1998), 227-249.

[28] _ Y. Long, The topological structures of $\omega$-subsets of symplectic groups, Acta Math. Sinica (English Series) 15 (1999), 255-268.

[29] B Bott formula of the Maslov-type index theory, Pacific J. Math. 187 (1999), $113-149$.

[30] systems, Third School on Nonlinear Analysis and Applications to Differential Equations (Oct. 12-30, 1998), ICTP Lecture Notes, SMR 1071/2, in Minimax Theory, Morse Theory and the Applications to Differential Equations (H. Brezis, S. Li, J.-Q. Liu, and P. Rabinowitz, eds.), Internat. Press, to appear.

[31] Precise iteration formulae of the Maslov-type index theory and ellipticity of closed characteristics, Adv. in Math. 154 (2000), 76-131.

[32] Y. Long and D. Dong, Normal forms of symplectic matrices, Acta Math. Sinica 16 (2000), $237-260$.

[33] Y. Long and E. Zehnder, Morse theory for forced oscillations of asymptotically linear Hamiltonian systems, in Stochastic Processes, Physics, and Geometry (S. Albeverio, et al., eds.), 528-563, World Sci. Publ., Teaneck, NJ (1990).

[34] Y. Long and C. ZHu, Maslov-type index theory for symplectic paths and spectral flow. I, Chinese Ann. of Math. 20 (1999), 413-424.

[35] Maslov-type index theory for symplectic paths and spectral flow. II, Chinese Ann. of Math. 21 (2000), 89-108.

[36] J. K. Moser, Periodic orbits near an equilibrium and a theorem by A. Weinstein, Comm. Pure Appl. Math. 29 (1976), 727-747.

[37] P. Rabinowitz, Periodic solutions of Hamiltonian systems. Comm. Pure Appl. Math. 31 (1978), 157-184.

[38] Minimax methods in critical point theory with applications to differential equations, CBMS Regional Conf. Ser. in Math. 65, A. M. S., Providence, RI, 1986.

[39] A. Szulkin, Morse theory and existence of periodic solutions of convex Hamiltonian systems, Bull. Soc. Math. France 116 (1988), 171-197.

[40] C. Viterbo, Indice de Morse des points critiques obtenus par minimax, Ann. Inst. H. Poincaré Anal. Non Linéaire 5 (1988), 221-225.

[41] - A new obstruction to embedding Lagrangian tori, Invent. Math. 100 (1990), 301-320.

[42] F. W. WARner, Foundations of Differentiable Manifolds and Lie Groups, Springer-Verlag, New York, 1983.

[43] A. Weinstein, Normal modes for nonlinear Hamiltonian systems, Invent. Math. 20 (1973), $47-57$.

[44] $\longrightarrow$ Periodic orbits for convex Hamiltonian systems, Ann. of Math. 108 (1978), $507-518$. 
[45] V. Yakubovich and V. Starzhinskit, Linear Differential Equations with Periodic Coefficients. 1,2, John Wiley \& Sons, New York (1975).

(Received May 13, 1999)

(Revised March 28, 2001) 